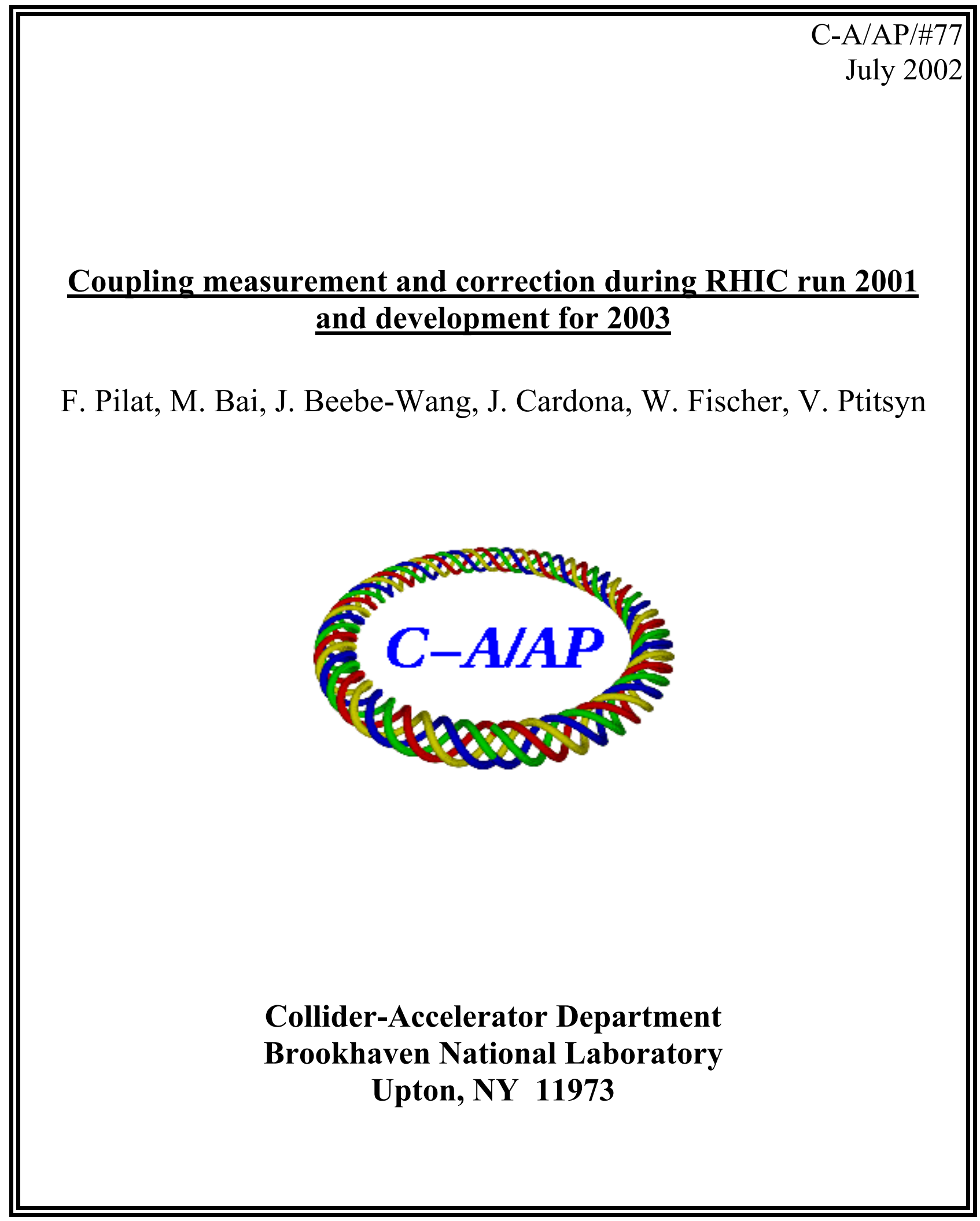




\title{
Coupling measurement and correction during RHIC run 2001 and development for 2003
}

\author{
F. Pilat, M. Bai, J. Beebe-Wang, J. Cardona, W. Fischer, V. Ptitsyn
}

1. Introduction

2. Local Interaction Region coupling correction

2.1 IR bumps method

2.2 Action-phase jump method

2.3 Local correction during run 2001

2.4 Comparison between beam-based and bench alignment measurements.

3. Global correction of coupling

3.1 Performance in run 2001

3.2 Global decoupling application package

4. Measurement of the coupling resonance

4.1 The method

4.2 Experiment and analysis

5. Plans and developments for coupling correction in 2003

\section{Introduction}

Coupling correction is essential for the operational performance of an accelerator, and RHIC is no exception. Effective independence of the transverse degrees of freedom makes measurements and diagnostics easier, and it is usually advantageous to operate an accelerator close to the coupling resonance to minimize nearby nonlinear sidebands. Besides these general considerations, the RHIC specific requirement of stringent tune control on the ramp (especially for polarized proton operations) demands good control of coupling effects, to ease the operation of the PLL based tune feedback system.

The coupling correction strategy that we used during the run 2001 is based on operational experience of other accelerators, simulation and studies on coupling effects performed during the RHIC design and construction phase, and, most relevant, the analysis of data and results gathered during the previous RHIC run in the year 2000 .

First, we used the independently powered skew quadrupole correctors embedded in the IR triplets to compensate locally the effect of roll alignment errors. The initial local corrector settings, based on the analysis of the run 2000 data, are applied at injection and ramped. The IR local coupling compensation techniques and the performance during the RHIC 2001 run are described in Section 2. In particular, results for the skew quadrupole strengths obtained with the IR bump technique are compared to the action-phase jump method and found in good agreement. After the end of the run, the alignment of selected individual triplet cold masses was revisited and the measured roll errors were found in good agreement with the roll inferred from the beam-based measurements.

The residual coupling in the machine, from arc magnets, experimental magnets (and in addition the Siberian snakes during the polarized proton run) has been corrected with two orthogonal families of skew quadrupoles by minimizing the tune separation, a well established operational technique to correct coupling globally in the machine. This technique was also used during run 2000. The improvement in 2001 was the development of a set of application scripts to aid the global decoupling operation by taking advantage of both the tune meter and the Schottky detector for tune measurements, and by allowing easier skew 
quadrupole family setting. The scripts and the performance of the global coupling correction system are discussed in Section 3.

Local and global decoupling were the typical operational tools used in Run 2001, but development work for upgraded coupling correction techniques started during beam experiment time in run 2001, that are the basis for new developments planned for the next run. Section 4 reports results obtained in the measurement of the coupling resonance and related data analysis with the SUSSIX analysis package. Finally, Section 5 discusses the requirements for coupling correction in the upcoming run 2003 and the strategy to fulfill them. Several methods were presented at the RHIC Retreat (March 2002) that have the potential of correcting coupling without moving the tunes, opening the possibility of coupling compensation during the ramp. We will discuss these techniques in more detail and try to assess their feasibility for the next RHIC run and beyond.

The RHIC coupling correction system is described in Figure 1, which shows the skew quadrupole system for the Blue ring. The same configuration is replicated for the Yellow ring.

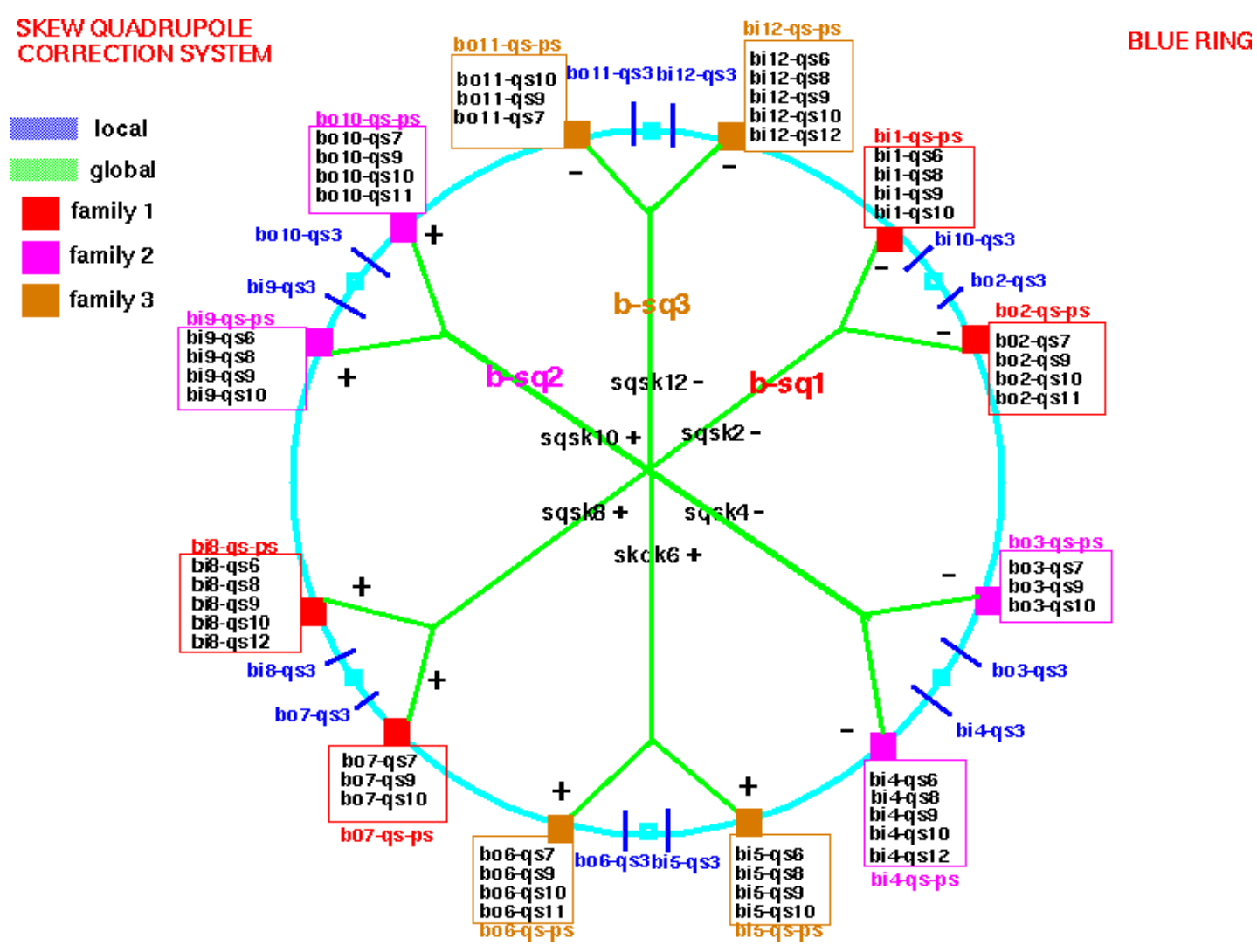

Figure 1. The skew quadrupole correction system in the RHIC Blue ring.

Each ring is equipped with 12 independently powered local skew quadrupole correctors located in the triplet corrector packages, and 48 skew correctors wired up in 3 families. The use of the 2 types of correctors is respectively discussed in Sessions 2 and 3.

\section{Local IR coupling correction}

During the RHIC run 2000 dedicated measurements were taken in the RHIC interaction regions (IR) to identify the coupling errors originating from rotation errors of quadrupoles of the IR triplets. Two techniques have been used independently. The first method, based on applying closed orbit bumps at the IR [1][2] triplets and looking at the rms closed orbit excited in the other plane, is described in Section 2.1. The second, which is called action-phase jump method [3], utilizes single dipole kicks to excite large beam 
trajectory oscillations and looks at the change of the amplitude (action) and phase of the trajectory oscillations in the other plane after every interaction region. The latter is described in Section 2.2.

\subsection{IR bump method}

Using three dipole correctors a closed orbit bump can be formed around any RHIC interaction region triplet. The maximum of the orbit excursion produced by the bump is located inside the triplet and in the absence of local gradient or skew errors the closed orbit in the rest of the ring would be undisturbed. If skew errors are present inside the triplet this is no longer the case. Closed orbit oscillations are excited in the plane orthogonal to the plane of the orbit bump.

Figure 2 shows a typical closed orbit bump in an interaction region. Closed bump data are obtained by taking the difference between orbits before and after setting the correctors for the requested bump. The bump settings are calculated by the on-line model, which assumes the ideal machine optics.

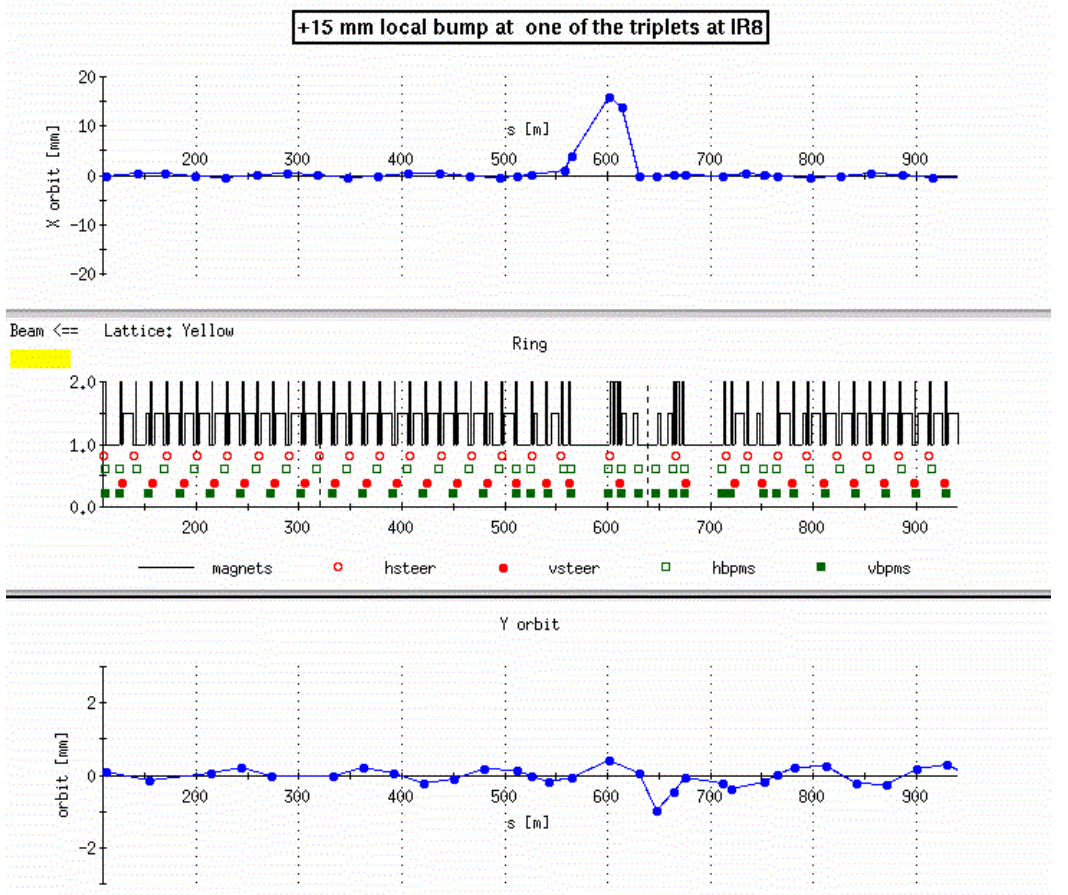

Figure 2. Example of interaction region (horizontal) orbit bump.

The amplitude of the closed orbit excitation is proportional to the bump amplitude and to the value of the coupling error. Since the RHIC rings have local skew quadrupole correctors inside every IR triplet (see Figure 1) and the betatron phase advance over the triplet is very small, corrector strengths can be calculated to eliminate this orbit excitation.

Let us consider a closed orbit bump applied in the horizontal plane. Defining the closed orbit bump amplitude $A_{\mathrm{x}}$ as the orbit excursion at the position of the horizontal dipole corrector in the triplet, the orbit excited in the vertical plane because of a coupling error $\kappa$ is:

$$
y=\frac{\sqrt{\beta_{y}}}{2 \sin \pi v_{y}} \frac{A_{x}}{\sqrt{\beta_{x}^{d c}}} \cos \left(\left|\Psi_{y}\right|-\pi v_{y}\right) \int_{\text {triplet }} \kappa \sqrt{\beta_{x} \beta_{y}} d s
$$

where $\Psi_{y}$ is the vertical betatron phase advance which changes with the betatron tune $v_{y}$.

$\beta_{x}^{d c}$ is the horizontal beta function at the position of the triplet horizontal dipole corrector. The effect of coupling is quantified by an integral which is taken over the quadrupoles of the given triplet. 
The orbit analysis is based only on data from beam position monitors in the arcs. The vertical beta function has the same value $(48 \mathrm{~m})$ at all arc vertical BPM locations. This simplifies the calculation of the orbit rms value which after averaging over the phase $\Psi_{y}$, is:

$$
y_{r m s}=\frac{\sqrt{\beta_{y}^{b p m}}}{2 \sqrt{2}\left|\sin \pi v_{y}\right|} \frac{A_{x}}{\sqrt{\beta_{x}^{d c}}} \int \kappa \sqrt{\beta_{x} \beta_{y}} d s=\lambda A_{x}
$$

where $\beta_{y}^{b p m}$ is taken at the arc BPM's.

Although $y_{r m s}$ is always considered as a positive mathematical value, in order to distinguish the sign of a coupling error we consider it as a signed value. Its sign is defined as the sign of the orbit data measured at a position in the arcs where, according to the machine optics, one expects a positive orbit measurement for a positive coupling error $\kappa$ and a positive bump amplitude $A_{\mathrm{x}}$.

Having $y_{r m s}$ and $A_{\mathrm{x}}$ from the measurements, the linear coefficient $\lambda$ can be extracted. One then calculates the IR skew quadrupole integrated corrector strength required to compensate for the coupling error. This is given by

$$
(k l)_{s c}=-\frac{\lambda}{\sqrt{\beta_{x}^{s c} \beta_{y}^{s c}}} \frac{2 \sqrt{2} \sqrt{\beta_{x}^{d c}}\left|\sin \pi v_{y}\right|}{\sqrt{\beta_{y}^{b p m}}}
$$

with $\beta_{x, y}^{s c}$ the beta functions at the skew corrector. Although we considered the effect of a horizontal orbit bump on the vertical orbit rms, switching the $\mathrm{y}$ and $\mathrm{x}$ indexes gives us the opposite case with a vertical bump and the horizontal orbit rms. Moreover, from the formulas listed above one can see that the coupling value error $\kappa$ as well as the skew corrector strength are weighted by a factor $\sqrt{\beta_{x} \beta_{y}}$ that is symmetric with respect to $\beta_{x}$ and $\beta_{y}$. Thus, whatever orbit bump is used for the experiment, horizontal or vertical, it has to lead to the same value of the skew corrector strength.

Figures 3 and 4 summarize the measured data of the rms orbit excited in the plane orthogonal to the bump plane as a function of the bump strength. Measurements were taken in 3 interaction regions (IR2, IR6, IR8) in both RHIC rings. For half of the triplets, measurements were done in both the horizontal and vertical planes but for the other half only horizontal bumps were used. The bump amplitude was varied from $5 \mathrm{~mm}$ to up to $50 \mathrm{~mm}$ in some triplets. For the larger bump amplitudes some of the curves deviate from linearity, a signature of nonlinear effects in the IR triplets

The coefficient $\lambda$ has been extracted by taking the linear coefficient of the curve polynomial fit. The corrector strengths have been calculated according to formula (3) and are listed in Tables 1 ("Closed orbit bumps" column) and 2 in Section 2.3. For triplets with both horizontal and vertical bump data, the skew corrector strength is taken as the average between the two measurements (plus and minus an error defined as a half of the difference between the two measurements). There is good agreement between horizontal and vertical bump measurements for all triplets except the BI1 triplet (Blue ring) where the error of 0.16 is comparable with the calculated average strength of -0.22 . Another uncertainty appeared at the YI6 (Yellow ring) triplet where the sign of the skew corrector could not be trustfully determined. 


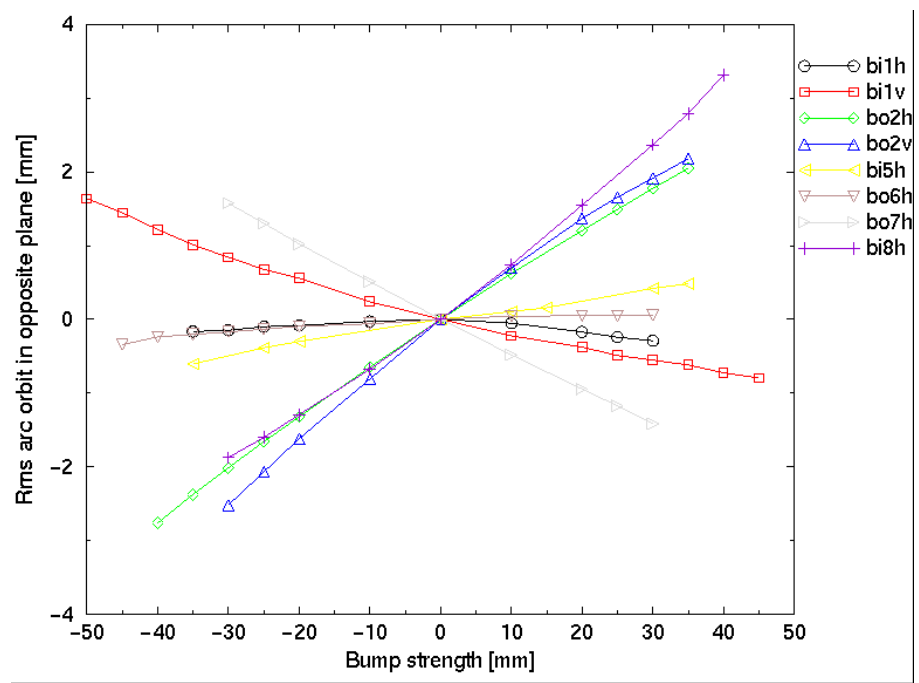

Figure 3. Orbit rms measured in the opposite plane versus bump strength (Blue Ring)

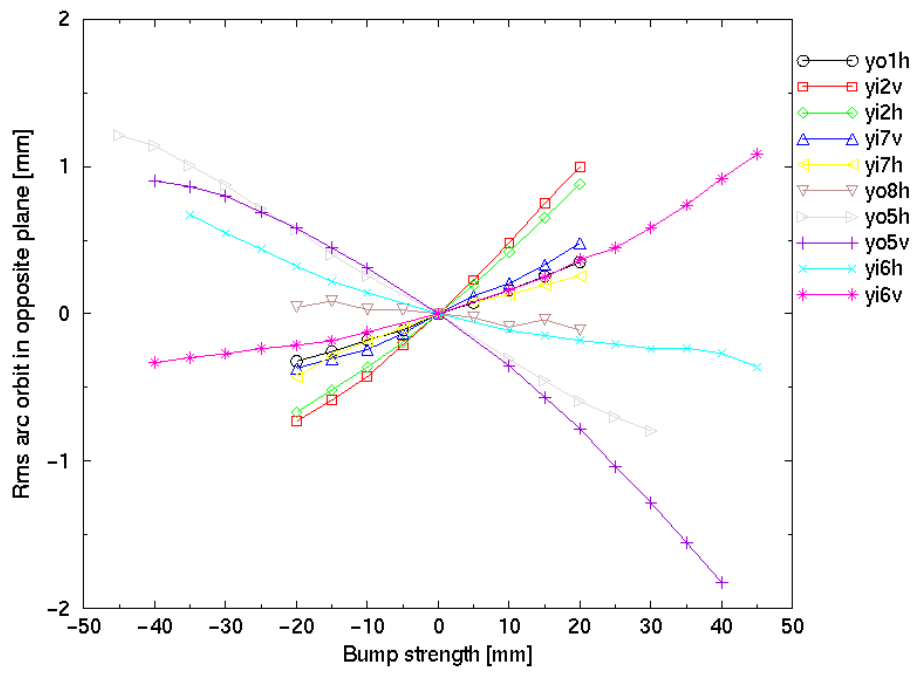

Figure 4. Orbit rms measured in the opposite plane versus bump strengths (Yellow Ring)

\subsection{Action-phase jump method}

Under ideal conditions, the action and phase of the betatron oscillations of a particle should remain constant all around the ring. Magnetic errors in the different elements of the ring can lead to a change of these two constants of motion, and these changes can be used to determine the location of the errors and their strengths. Action and phase associated with RHIC particle orbits at particular positions in the ring are obtained from pairs of adjacent BPM measurements. BPM measurements are converted into action and phase after inverting the equations:

$$
\begin{aligned}
& x_{1}=\sqrt{2 J \beta_{1}} \sin \left(\psi_{1}-\varphi\right) \\
& x_{2}=\sqrt{2 J \beta_{2}} \sin \left(\psi_{2}-\varphi\right)
\end{aligned}
$$

where $x_{1}$ and $x_{2}$ correspond to any two adjacent BPM measurements, $\beta_{1}, \beta_{2}, \psi_{1}, \psi_{2}$ their corresponding beta functions and phase advances and finally $J$ and $\varphi$ are the action and phase. Equation 4 is applied to all adjacent BPM measurements in the ring to obtain action and phase as a function of the longitudinal position s. During the RHIC 2000 run, studies of action and phase confirmed the existence of significant coupling 
errors in the IR's. A method based on first turn measurements and action and phase analysis was developed to quantify the coupling error and correct for it. The positive results obtained from the previous studies stimulated the extension to a more general method that can evaluate, from closed orbits (RHIC 2001 run) not only skew errors but also gradient and nonlinear error in the RHIC IR's.

\subsubsection{Experimental procedure}

Action and phase studies require stable and defined orbit betatron oscillations. Such betatron oscillations can be produced at RHIC by simply turning on a dipole corrector. The corrector must be chosen such that the phase advance between the corrector and the interaction region under study is close to an odd integer of $\pi / 2$. This condition guarantees that the orbit will be near to its maximum when going trough the interaction region. The strength of the corrector is a trade-off, strong enough to cause a large betatron oscillation but weak enough to avoid beam losses. In RHIC, a few tenths of mrad produce large enough oscillations without compromising the beam.

The resulting orbit however arises not only from the dipole corrector intentionally turn on but also has small contributions from dipole errors always present in the ring. To eliminate such contributions, the baseline (orbit when the dipole corrector is off) is subtracted from the original orbit producing what is called a difference orbit. This procedure also eliminates possible systematic errors associated with the BPM measurements.

In principle, betatron oscillations should be present in only the plane where the dipole corrector is turn on. However, due to coupling, betatron oscillations are also present in the plane opposite to the plane where the dipole corrector is turned on. The betatron oscillations in the plane opposite to the plane of the acting dipole corrector are the ones that will be analyzed since they give a direct indication of the amount of coupling present in the ring.

\subsubsection{Analysis}

Equations 4 are applied to the difference orbit to get functions of action and phase like the ones shown in Figure 5. To obtain this particular plot, the vertical dipole corrector bo7-tv7 was set to $0.23 \mathrm{mrad}$, the induced orbit in the opposite plane measured (top rectangle of Figure 5) and then action and phase obtained (middle and bottom rectangles of Figure 5) by repeatedly using formulas 4 .

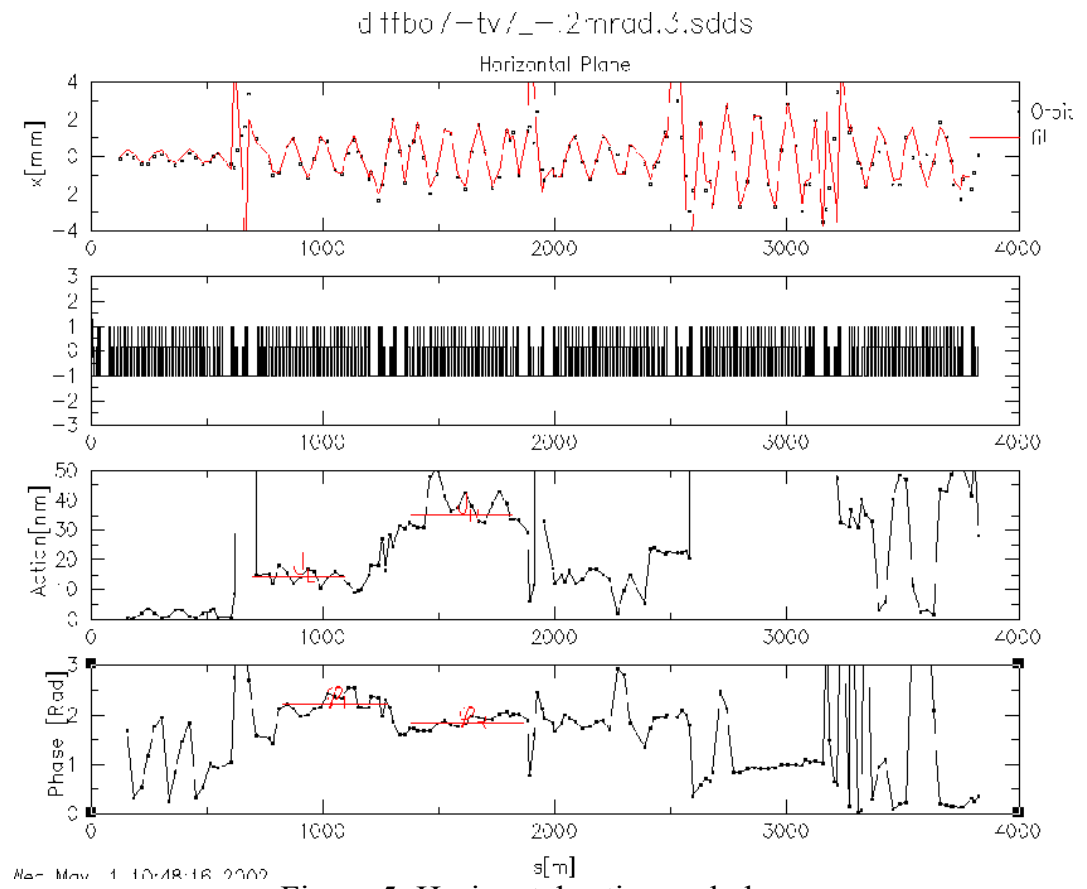

Figure 5: Horizontal action and phase 
Figure 5 suggests that action and phase remain constant in the arcs while making significant jumps at the interaction regions. The analysis of first turn orbits indicates that the jumps at the IR' $s$ are mainly due to the local skew quadrupole errors. All RHIC IR triplet correction packages include 1 skew quadrupole layer (see Figure 1). The local skew correction is aimed at eliminating the betatron oscillations generated by the skew errors at the triplet, or in mathematical terms:

$$
\left\lfloor\sqrt{2 J_{x}^{t r i p}}+(k l)_{s c} y^{s c} \sqrt{\beta_{x}^{s c}}\right\rfloor \sqrt{\beta_{x}(s)} \sin \left(\psi_{x}(s)-\varphi_{x}^{t r i p}\right)=0
$$

where $J_{x}^{\text {trip }}$ is the action associated with the betatron oscillations originated by the skew quadrupole errors at the triplet, $y^{s c}$ is the beam position in the plane of kick (in this case the vertical plane) at the location of the skew quadrupole corrector, $\beta_{x}^{s c}$ is the beta function at the position of the skew quadrupole corrector, $(k l)_{s c}$ is the strength of the corrector, $\varphi_{x}^{t r i p}$ the phase at the triplet and $\beta_{x}(s)$ and $\psi_{x}(s)$ the beta functions and phase functions. $(k l)_{s c}$ trivially follows from Equation 5.

$$
(k l)_{s c}=-\frac{\sqrt{2 J_{x}^{t r i p}}}{y^{s c} \sqrt{\beta x^{s c}}}
$$

Since the two skew quadrupole correctors of a specific IR have a difference phase advance close to $\pi$, only one skew quadrupole corrector can be used to compensate the skew errors in the whole IR. This is specially useful when is not possible to have individual estimates of $J_{x}^{\text {trip }}$ for the left and right triplets but rather a integrated value $J_{x}^{I R}$ for the whole IR, as it was here experimentally the case. It is possible to show that formula 6 still holds if $J_{x}^{t r i p}$ is changed by $J_{x}^{I R}$.

If the action in the arc immediately to the left (Blue Ring) of the IR under study is zero (see Figure 5) the action immediately to the right is equal to $J_{x}^{I R}$. Strengths are then trivially calculated using Formula 6 . We called the strengths so obtained principal strengths. On the other hand, if the action in the arc immediately to the left of the IR under study is different from zero an additional step is required to obtain $J_{x}^{I R}$ :

$$
J_{x}^{I R}=J_{x}^{R}+J_{x}^{L}-2 \sqrt{J_{x}^{L}} \sqrt{J_{x}^{R}} \cos \left(\varphi_{x}^{R}-\varphi_{x}^{L}\right)
$$

here, $J_{x}^{L}$ is the action on the left hand side of the IR, $J_{x}^{R}$ is the action on the right hand side and $\varphi_{x}^{L}$ and $\varphi_{x}^{R}$ are the corresponding phases. We call the strengths thus obtained secondary strengths. Figure 5 shows how actions and phases are labeled when strengths are calculated at IR10. In principle, only one orbit would be required to extract the corrector strength required at a particular interaction region. However, several orbits produced by different dipole correctors and different strengths are used to estimate the uncertainty in the measurement.

\subsubsection{Results}

The different measurements of principal strengths for a particular interaction region are, in general, very consistent. In other words, the uncertainty associated with principal strengths is very small. For almost all interactions regions the uncertainties associated with secondary strengths are bigger than the ones associated with principal strengths.

The difference in the uncertainties of principal and secondary strengths might be due to the presence of gradient errors. Their magnitudes can be very different in the 2 cases. The difference lies in the fact that the beam position at the interaction region of orbits that are used to calculate principal strengths is very small when compared with the beam position at the same interaction region of orbits that are used to calculate secondary strengths. Since gradient errors at the interaction region depend linearly on the position of the beam, the difference between the gradients errors in each case can be also very different. The "action-phase jump" column in the Table 1 (Section 2.3) shows the estimated principal strengths obtained from the analysis of 40 difference orbits taken during RHIC run 2000 in the Blue ring. 


\subsection{Calculated corrector strength}

The resulting IR skew corrector strengths, using the two methods described, are summarized in Table 1 and 2. For the Blue ring, where we have calculations obtained from both methods, we compare the results of the methods for three interaction regions. Because the action-phase jump technique determines the corrector strength for whole interaction region, it should be compared with the sum of both triplet corrector strengths calculated by closed orbit bumps, for a given IR. The agreement between results is good for all three measured interaction region

\begin{tabular}{|c|c|c|c|}
\hline \multirow{2}{*}{ IR } & \multirow{2}{*}{ Triplet } & \multicolumn{2}{|c|}{ Calculated corrector strengths $\left(10^{-3} \mathrm{~m}^{-1}\right)$} \\
\hline & & Closed orbit bumps & Action-phase jump \\
\hline IR2 & $\begin{array}{l}\mathrm{BI} 1 \\
\mathrm{BO} 2\end{array}$ & $\begin{array}{c}-0.22+/-0.16 \\
1.23+/-0.15\end{array}$ & $0.99+/-0.14$ \\
\hline IR4 & & & $0.63+/-0.06$ \\
\hline IR6 & $\begin{array}{l}\mathrm{BI5} \\
\mathrm{BO6}\end{array}$ & $\begin{array}{l}0.39 \\
0.12\end{array}$ & $0.60+/-0.15$ \\
\hline IR8 & $\begin{array}{l}\mathrm{BO} 7 \\
\mathrm{BI} 8\end{array}$ & $\begin{array}{l}-0.84 \\
1.32 \\
\end{array}$ & $0.67+/-0.07$ \\
\hline IR10 & & & $1.00+/-0.15$ \\
\hline IR12 & & & $0.18+/-0.03$ \\
\hline
\end{tabular}

Table 1. Calculated IR skew corrector strengths for the Blue ring.

\begin{tabular}{|c|c|c|}
\hline IR & Triplet & $\begin{array}{ll}\text { Calculated } & \text { corrector } \\
\text { strength }\left(10^{-3} \mathrm{~m}^{-1}\right) & \\
\end{array}$ \\
\hline IR2 & $\begin{array}{l}\text { YO1 } \\
\text { YI2 }\end{array}$ & $\begin{array}{l}0.3 \\
0.76+/-0\end{array}$ \\
\hline IR6 & $\begin{array}{l}\text { YO5 } \\
\text { YI6 }\end{array}$ & $\begin{array}{r}-0.94+/-0.08 \\
+/-0.36+/-0.04 \\
\end{array}$ \\
\hline IR8 & $\begin{array}{c}\text { YI7 } \\
\text { YO8 }\end{array}$ & $\begin{array}{l}0.36+/-0.04 \\
-1.1\end{array}$ \\
\hline
\end{tabular}

Table 2. Calculated IR skew corrector strengths for Yellow ring (closed orbit bumps data only)

\subsection{Local correction in run 2001}

The two methods described in the previous section and data from run 2000 provided us with a set of skew corrector strengths (Tables 1 and 2), but not a complete set. We had no data for all Yellow triplets in IR10, IR12 and IR4, so the local correction had to be completed and tested at the beginning of the 2001 run.

\subsubsection{Local IR correction during operations}

The run 2001 optics was different from the one in run 2000. The injection $\beta^{*}$ in 2001 was $10 \mathrm{~m}$ at all interaction points. We could then expect a decreased effect of the coupling error at injection because of the lower $\beta$ function in the IR triplets. On the other hand, the collision optics at top energy in 2001 used $\beta *$ of 1 and 2 meters, which, left uncompensated, would have considerably enhanced the coupling effect. Therefore we planned to put the calculated IR corrections early into the run, before globally decoupling with the skew families in the arcs. (Ssee Section 3). 


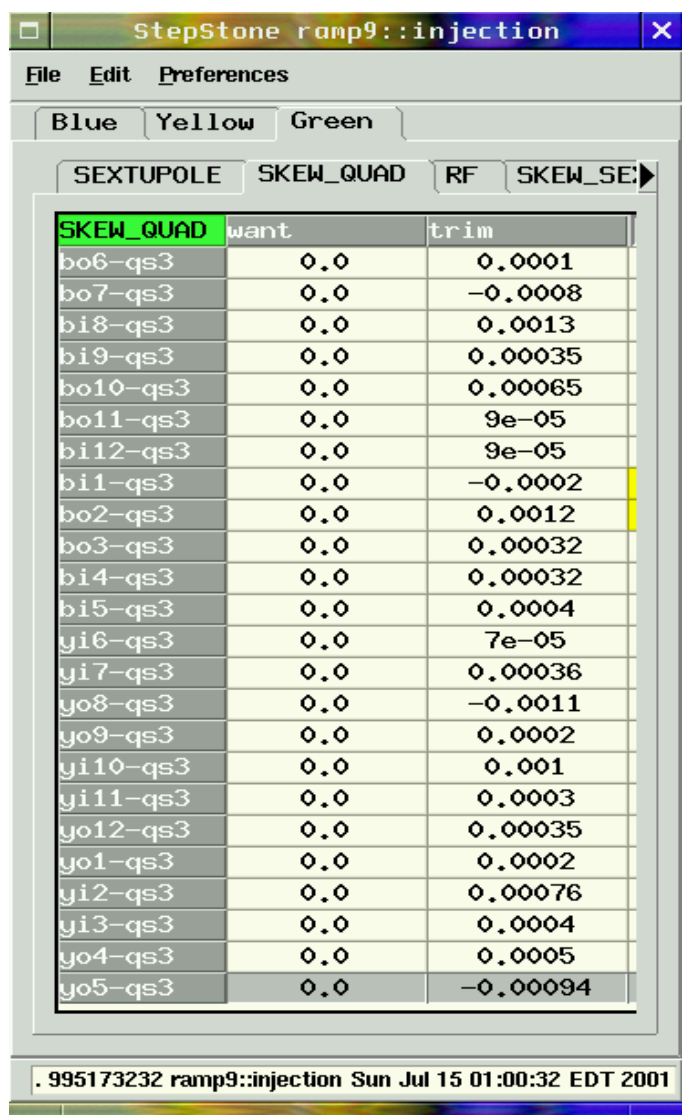

Figure 6. Setting for the IR skew quadrupole correctors in 2001 (Blue and Yellow)

The local IR skew corrector set, activated in the machine in early July, is shown in Figure 6 from the RampEditor. Most of the triplets were corrected using the calculated corrector values. For the triplets where we had only predictions from the action-phase jump method for a whole interaction region, the strength has been divided equally over the two correctors in the same IR.

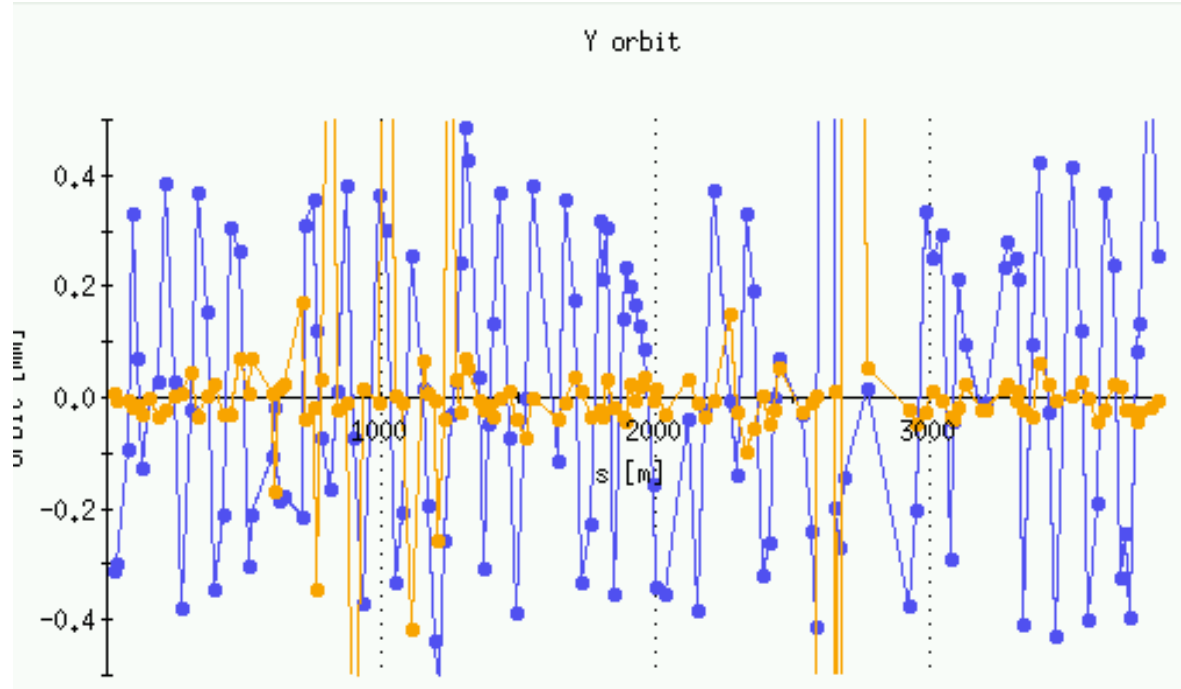

Figure 7. The difference orbit before (blue) and after (orange) skew correction at the YO10 triplet. The vertical orbit was excited by applying a $10 \mathrm{~mm}$ amplitude horizontal bump. 


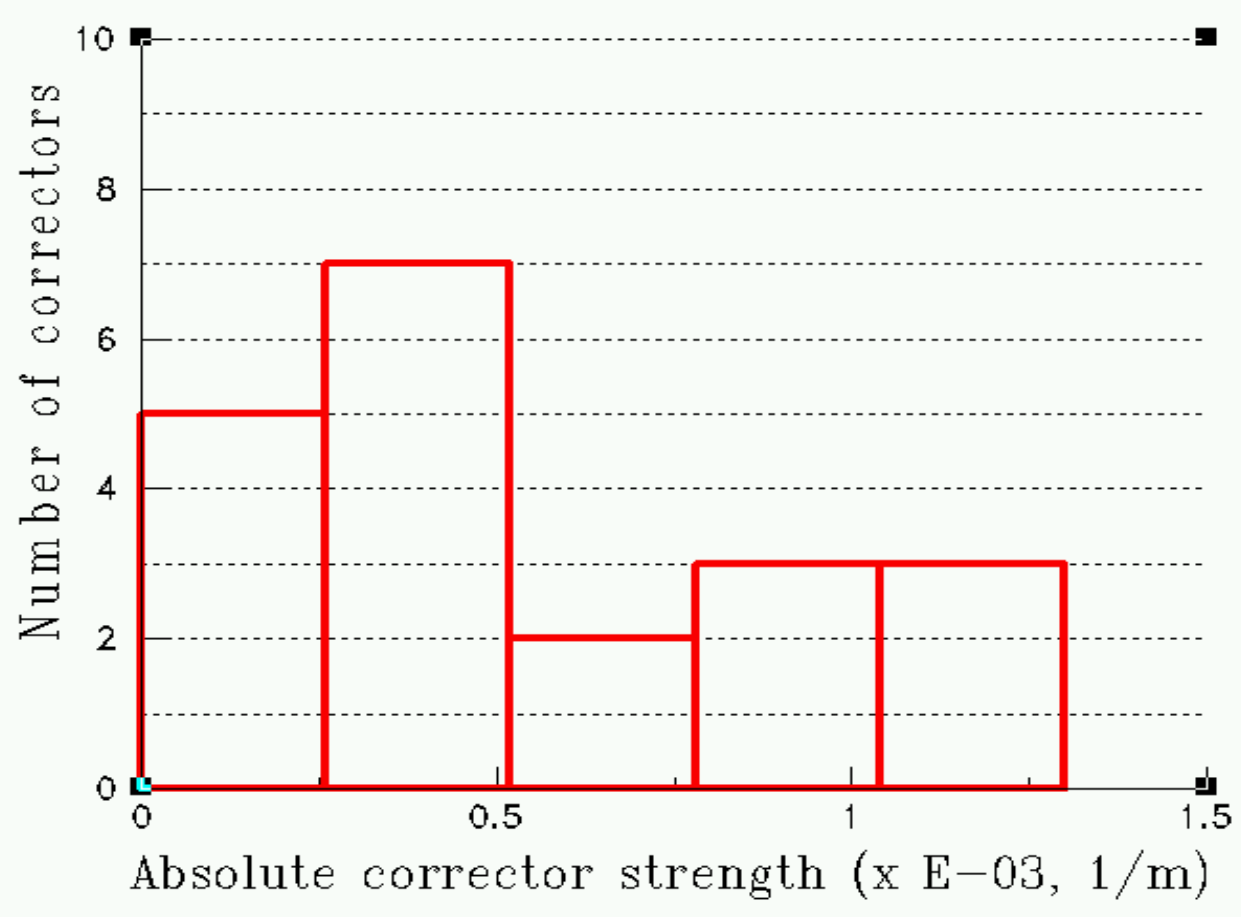

Figure 8. Distribution of skew corrector strengths.

For the Yellow triplets where we did not have calculated corrector strengths from run 2000, the correction was done online, operationally, via closed IR bumps. Figure 7 shows the IR correction done with a closed bump in the YI10 triplet. Vertical orbit oscillations excited by applying a horizontal orbit bump of $10 \mathrm{~mm}$ amplitude were damped by using the local skew corrector. For most of the regions where the calculated corrector strength had been applied, we tested the correction quality with closed orbit bumps. That demonstrated that the calculated correction works very well. Only at the YO1 and YI6 triplets the corrector strength required some adjustment from the calculated values. Figure 8 shows a distribution of absolute skew corrector strengths. The largest corrector strengths were needed at BI8, BO2 and YO8 triplets. The quadrupole roll error of the triplets as the main source of the skew IR error is discussed in Section 2.4.

The strength required for the bi8-qs3 corrector, corresponding to $49 \mathrm{~A}$ in the power supply, is beyond the recommended current limit of $47.5 \mathrm{~A}$. To limit the current in bi8-sq3, the correctors were readjusted at the flat-top stone by distributing some strength to the corrector across the IP (bo7-qs3). In order to insure the locality of the correction, the plan for run 2003 is to increase the power supply limit to 55A for the correctors running close to the limit.

Figure 9 shows the local corrector strengths, blue and yellow respectively, over the length of the run (including polarized proton operations).

Starting from early July the correction was fairly stable, with the exception of a few power supply trips. The effect of a tripped power supplies was normally compensated by adjusting the corrector at the other triplet while keeping the sum of the two IR corrector strength constant. In particular, the corrector at 1 o'clock in Blue had to compensated by the 2 o'clock one for most of the run. 

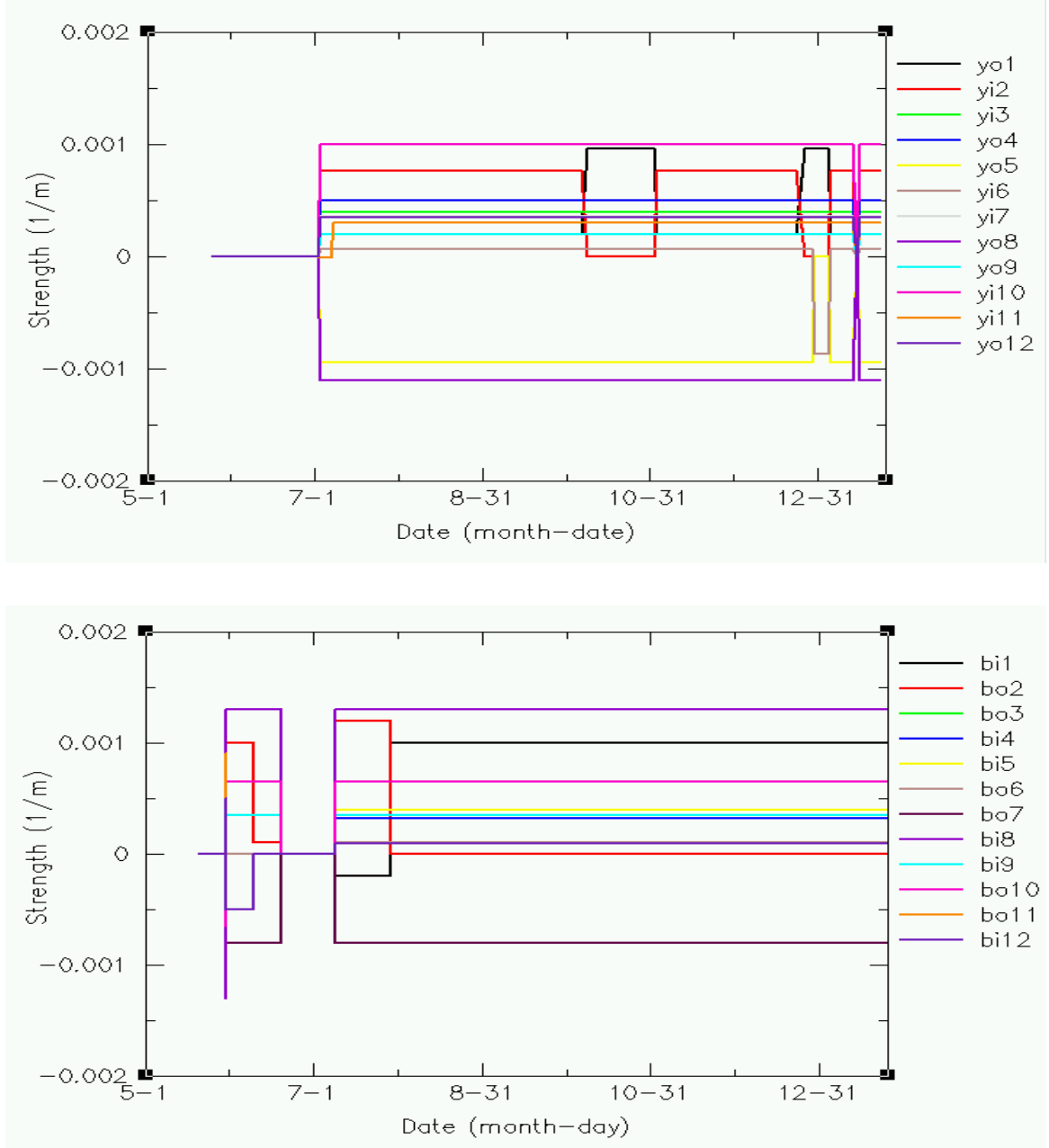

Figure 9. Yellow and blue skew quadrupole IR settings during Run 2001.

\subsubsection{Extension of the action-jump method}

In the 2001 run the action-phase jump technique described in Section 2.2 was extended to add the analysis of gradient and nonlinear errors in the IR's to the original coupling errors. After a brief introduction on the experimental procedure, we will discuss here only the coupling results.

Closed orbits are obtained from the average of many thousands of turns. This leads to small uncertainties in the beam position monitor measurements, which in turns leads to a more precise determination of the magnetic errors when compare with first turn orbits measurements. However, closed orbits can be used almost exclusively to find secondary strengths (see Section 2.2) and the determination of secondary strengths might be affected by the presence of significant gradient errors. This is especially true when the measurements are repeated after the correctors are set. In that case, the skew quadrupole errors are very close to zero and the gradient errors can be significantly larger, which in turn will lead to a false measurement of the secondary strength. Principal and secondary strengths can also be affected by the presence of non-linear errors. All this motivated the development of a general method that is able to discriminate between gradient errors, skew quadrupole errors and non-linear errors.

In this case, the experiment described in section 2.2.1 is repeated four times for each dipole corrector, for 4 different corrector strengths. The measured quantities are closed orbits and this implies that both horizontal and vertical orbits must be analyzed, and also that the resolution of the measurements is better. In particular, the 2 DX BPM' s can be used to calculate action and phase in the region between triplets and to independently estimate errors at each triplet. 
In this case the action and phase jump can be described as if it were coming from a general magnetic kick $\Delta^{\text {trip }}$ that can be obtained from measured quantities:

$$
\Delta x^{t \text { trip }}=\sqrt{\left(J_{x}^{L}+J_{x}^{R}-2 \sqrt{J_{x}^{L} J_{x}^{R} \cos \left(\psi_{x}^{L}-\psi_{x}^{R}\right)}\right.} / \sqrt{\beta_{x}^{\text {trip }}}
$$

where $L$ and $R$ label action and phases at the left and right hand side of the triplet under study. The $\Delta x$,trip thus obtained is an effective kick that we can assume produced at a particular location $s_{o}$ within the triplet. $s_{o}$ can be chosen arbitrarily and it determines the beta function $\beta_{x}^{\text {trip }}$ to be used in the previous formula.

Choosing $s_{o}$ exactly at the location of the skew quadrupole corrector simplifies the determination of the corrector strengths needed to compensate for the errors. On the other hand, $\Delta x^{\text {ttrip }}$ corresponds to the sum all possible error present at a particular triplet, skew quadrupole, gradient or non linear errors. In general, $\Delta x^{\prime}$ can be written as:

$$
\Delta x^{\prime}=-\frac{B_{y} l}{B_{\rho}}
$$

where $B_{y}$ is the vertical component of the magnetic field error, $l$ is the total length of the magnet and $B_{\rho}$ is the rigidity. A similar equation can be written for a vertical kick. By expanding $B_{x}$ and $B_{y}$ in their magnetic multipole components [4], we can see that:

$$
\begin{aligned}
& \Delta x^{\prime}=\left(A_{1} y_{o}-B_{1} x_{o}+2 A_{2} x_{o} y_{o}+B_{2}\left(-x_{o}^{2}+y_{o}^{2}\right)+\ldots\right) \\
& \Delta y^{\prime}=\left(A_{1} x_{o}+B_{1} y_{o}+2 B_{2} x_{o} y_{0}+A_{2}\left(x_{o}^{2}+y_{o}^{2}\right)+\ldots\right)
\end{aligned}
$$

Here the A's and B's are the triplet magnetic multipoles. $A_{l}$ is the skew quadrupole error, $B_{I}$ to the gradient error, $x_{o}$ and $y_{o}$ the horizontal and vertical position of the beam at $s_{o}$. Since $s_{o}$ is chosen at the corrector location, from $A_{l}$ we can determine the skew corrector strength. It is possible to evaluate the different multipole components in equations 10 by taking several sets of delta measurements of given horizontal and vertical beam positions. In section 2.2 difference orbits were created with a dipole corrector set to different strengths. The magnetic kick at each triplet will vary as the strength of the corrector is varied. The position of the beam in both planes at the specific location $s_{o}$ will vary as well with the variations on the dipole corrector strength. As was mention in section 2.2 a set of four different orbits were taken for each dipole corrector. These orbits provide four points to be used to evaluate the coefficients in Equation 10.

The values of $\Delta x^{\prime}$ and $\Delta y^{\prime}$ as a function of the two variables $x_{o}$ and $y_{o}$ can be extracted from the difference orbits. $x_{o}$ and $y_{o}$ are no longer independent variables. The relation between these variables depends on the amount of coupling present in the ring and that can be easily determined experimentally. This means that $\Delta x^{\prime}$ and $\Delta y^{\prime}$ can be regarded as functions that depend only on one variable, $x_{o}$ or $y_{o}$

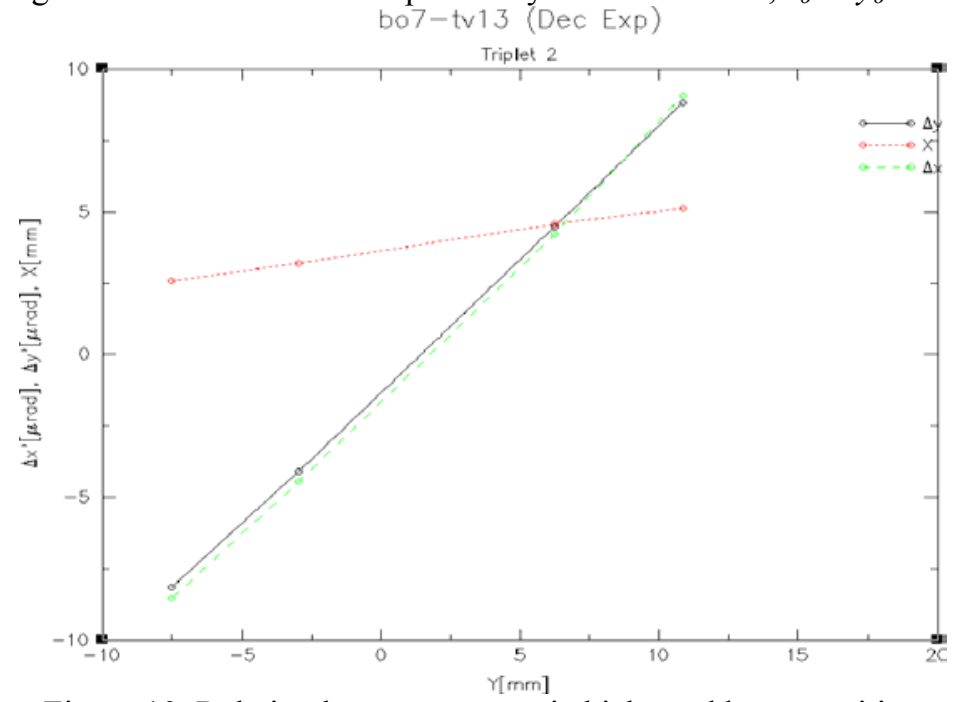

Figure 10. Relation between magnetic kicks and beam position

Figure 10 shows $\Delta x^{\prime}$ (green line), $\Delta y^{\prime}$ (black line) and $x_{o}$ as a function of $y_{o}$ at the right triplet of IR2 for a set of four difference orbits taken by turning on the vertical dipole corrector bo 7 -tv13 at strengths -0.1 
mrad, $-0.050 .05 \mathrm{mrad}$ and $0.1 \mathrm{mrad}$ (each point in the graph correspond to one strength). The behavior of $\Delta x^{\prime}$ and $\Delta y^{\prime}$ as function of $x_{o}$ is almost linear, that is, the contribution of nonlinear errors to the action and phase jump at the right triplet of IR 2 is very small. Comparing Equation 10 (only linear terms) with the slopes of the curves of Figure 10, it is possible to estimate the value of the linear errors $A_{l}$ and $B_{l}$ using:

$$
\begin{aligned}
& A_{1}=\left(C_{x}+C_{y} k\right) /\left(1+k^{2}\right) \\
& B_{1}=\left(C_{y}+C_{x} k\right) /\left(1+k^{2}\right)
\end{aligned}
$$

where $C_{x}$ is the slope of the curve $\Delta x^{\prime}$ vs. $y_{o}, C_{y}$ is the slope of the curve $\Delta y^{\prime}$ vs. $y_{o}$ and $k$ is the slope of the curve $x_{o}$ vs. $y_{o}$ The experiment is repeated with many other correctors and in different triplets, with results that are summarized in Table 3. The value of the skew quadrupole corrector corresponds is the average of the values obtained with the different dipole correctors. The large errors at the $2 \mathrm{o}$ 'clock triplets reflect the fact that the $\mathrm{BO} 2$ skew corrector was tripped and its strength was added to the BI1 corrector to provide the compensate.

\begin{tabular}{|c|c|}
\hline Triplet & Skew error $\left(10^{-3}\right)$ \\
\hline BO7 & $-0.001+/-0.01$ \\
\hline BI8 & $0.1+/-0.07$ \\
\hline BI9 & $-0.04+/-0.03$ \\
\hline BO10 & $0.13+/-0.03$ \\
\hline BI1 & $-1.11+/-0.03$ \\
\hline BO2 & 1 \\
\hline
\end{tabular}

Table 3. Measured Blue IR skew quadrupole errors (run 2001)

The errors limit the minimum skew error that can be resolved to $\sim 10^{-4} 1 / \mathrm{m}$. That many of the skew errors fall around or below this number was to be expected since the local skew correctors were already on when the data in Table 3 were taken. The magnitude of the error depends more on the dipole corrector that is chosen to produce the betatron oscillations than on the BPM measurements. This is an indication that the measurements are sensitive to the difference of phase advance between the dipole corrector and the IR. Even though the orbits used to find the errors at a particular IR were chosen with optimal phase advanced in one of the planes, it was not always possible to fulfill the same condition in the other plane. It is possible though to have a complete control over this problem if a horizontal and a vertical dipole corrector are used simultaneously to produce the betatron oscillation.

Another possible source of error is the slight difference in tunes between the model used to do the analysis and the real tune of the machine. This difference creates a slight slope in the graphs of phase vs. $s$ in the arcs. A retuning of the lattice model would hopefully reduce this tilt in the phase. After correction of these effects, it is not unreasonable to expect measurements of skew errors with significant figures up to $10^{-5}$ $1 / \mathrm{m}$.

\subsection{Comparison between beam-based and bench alignment measurements}

Table 4 summarizes the relations between the different beam-based measurements done at RHIC

\begin{tabular}{|c|c|c|c|c|c|c|}
\hline triplet & corrector & residual 2001 & total triplet & total IR & Orbit bump 2000 & action-phase \\
\hline BO7 & -0.8 & 0 & -0.8 & \multirow{2}{*}{0.6} & -0.84 & \multirow{2}{*}{0.67} \\
\cline { 1 - 3 } BI8 & 1.3 & 0.1 & 1.4 & & 1.32 & \multirow{2}{*}{1} \\
\hline BI9 & 0.35 & -0.035 & 0.32 & \multirow{2}{*}{1.1} & & \multirow{2}{*}{0.99} \\
\hline BO10 & 0.65 & 0.13 & 0.78 & & -0.22 & 1.23 \\
\hline BI1 & 1 & -1.1 & -0.1 & \multirow{2}{*}{0.9} & & \\
\hline BO2 & 0 & 1 & 1 & &
\end{tabular}

Table 4. Skew error measurements, comparison 2000 and 2001 (Blue). All values are given in 10 $1 / \mathrm{m}$.

The first column shows the triplet skew quadrupole corrector settings during RHIC run 2001. The second shows the measurement of the skew errors done during RHIC run 2001 with the action-phase jump method. 
This is effectively the residual error as the data were taken with the local correctors already on. The total triplet column lists the sum of the previous two quantities. The total IR column is the sum of the left and right triplets for each IR, from the previous column. The total triplet column and the total IR column allow a direct comparison with run 2000 measurements, where data were taken with correctors off. The orbit bump column shows the measurements of the skew errors done during the run 2000 with the orbit bump method, in good agreement with the 'total triplet' column. Two other columns to compare are the actionphase jump column and the total IR column. The action-phase jump column corresponds to the measurement done in 2000 with action-phase jump. The agreement is good in this case too. The beambased measurements evidenced that there is indeed a large coupling effect in the RHIC triplets. This motivated bench alignment measurements on selected triplets during the shutdown 2002.

The roll angles of individual cold masses inside the triplet were measured and the bench measurements compared with the beam-based predictions in Table 5 below. The roll angles are shown as if they were measured from the Lead End of the Q1 quadrupole. Roll angles can be easily related to the equivalent skew quadrupole error of a particular triplet by the formula:

$$
(k l)_{s c}=\frac{\sum_{i=1}^{3}\left(-2 \frac{\phi_{i}}{f_{i}}\right) \sqrt{\beta_{x}^{i} \beta_{y}^{i}}}{\sqrt{\beta_{x}^{\text {trip }} \beta_{y}^{\text {trip }}}}
$$

where $f_{i} \phi_{i} \quad \beta_{x}^{i}$ and $\beta_{y}^{i}$ correspond respectively to the focal lengths, roll angles, beta functions (in both planes) of each quadrupole in the triplet. $\beta_{x}^{\text {trip }}$ and $\beta_{y}^{\text {trip }}$ are the beta functions at the place where the equivalent skew quadrupole error is to be calculated, in this case at the position of the skew quadrupole corrector.

\begin{tabular}{|l|c|c|c|c|c|c|c|}
\hline Triplet & $\begin{array}{l}\text { Q1 } \\
\mathrm{mrad}\end{array}$ & $\begin{array}{l}\text { Q2 } \\
\mathrm{mrad}\end{array}$ & $\begin{array}{l}\text { Q3 } \\
\mathrm{mrad}\end{array}$ & $\begin{array}{l}\boldsymbol{k l} \\
10^{-3} 1 / \mathrm{m}\end{array}$ & $\begin{array}{l}\text { Orbit bump } \\
10^{-3} 1 / \mathrm{m}\end{array}$ & $\begin{array}{l}\text { Action-phase } \\
10^{-3} 1 / \mathrm{m}\end{array}$ & $\begin{array}{l}\text { Q2 roll eq. } \\
\mathrm{mrad}\end{array}$ \\
\hline Blue sector 8 & -5 & 2 & -0.7 & 1.6 & 1.3 & 1.4 & 3.7 \\
\hline Yellow sector 8 & -1.08 & 1.54 & -2.12 & -1.13 & -1.1 & & 2.9 \\
\hline
\end{tabular}

Table 5. Roll angles, comparison between beam based and alignment measurements.

Using the measured rolls (Table 5) and the design values for the focal lengths and the beta functions in equation 13, we find that the equivalent skew quadrupole error for triplet 8 in the Blue Ring is $1.6 \mathrm{e}^{-3} 1 / \mathrm{m}$ compared to $1.4 \mathrm{e}^{-3}$ (action-phase jump value in Table 4) and $1.3 \mathrm{e}^{-3}$ (orbit bump value in Table 4). Similarly, for the Yellow Ring the calculated value is $-1.13 \mathrm{e}^{-3} 1 / \mathrm{m}$ as compared to the measured value of $1.1 \mathrm{e}^{-3} \mathrm{1} / \mathrm{m}$. Taking into account that the measurement errors are about $10 \%$, there is excellent agreement between the strength derived from the measured roll angles and the strength derived from orbit based measurements.

\section{Global coupling correction}

A common operational way to correct global coupling in an accelerator is the minimum tune separation technique. This has been used at a variety of accelerators and at RHIC as well. It can be derived that the minimum achievable separation of the horizontal and vertical tune $\left(\Delta Q_{\text {min }}\right)$ in presence of coupling is given by:

$$
\Delta Q_{\min }=\frac{1}{\pi} \frac{\sqrt{\operatorname{det} E}}{\sin 2 \pi Q_{x}+\sin 2 \pi Q_{y}} \approx \frac{1}{2 \pi} \frac{\sqrt{\operatorname{det} E}}{\sin 2 \pi Q_{o}} \quad \begin{aligned}
& Q_{x}=Q_{o}+\Delta / 2 \\
& Q_{y}=Q_{o}-\Delta / 2
\end{aligned}
$$

where $\mathrm{E}$ is the $2 \times 2$ off diagonal coupling matrix. For $N$ skew quadrupoles of strength $k$ and length $L$ wired into a family, neglecting beta and phase differences, we can write the following relation:

$$
\Delta Q_{\min } \approx \frac{N}{2 \pi} k L \sqrt{\beta_{x} \beta_{y}}
$$


The operational way to correct coupling is to reach the minimum separation via a tune scan to bring the tunes together. At the minimum, a pair of orthogonal skew quadrupole families is varied to reduce the separation, ideally limited only by the resolution of the tune measuring system. Figure 1 describes the schematics of the skew quadrupole families in the RHIC Blue ring (the Yellow ring configuration is the same). Every family consists of 16 skew quadrupoles, powered by 4 power supplies that are hardware-wise independent but are set at the same strength in software, at the RampEditor level. (In fact, given the phase advance in the lattice, the supplies of the family are powered anti-symmetrically). There are 3 families in each ring, given the lattice symmetry, and the phase between families if approximately 120 degrees. It is easy to verify that the pair (fam1, (fam2-fam3) is orthogonal. The effect of different coupling sources and the capabilities of the correction system itself have been simulated during the design phase and verified with measurements during the RHIC runs 2000 and 2001. Table 6 lists coupling effects in term of their effect on the RHIC $\Delta Q_{\min }$ obtained in simulation and the capability of the correction system, and compares them with actual measurements during RHIC runs.

\subsection{Global coupling correction performance 2001}

The global coupling correction system has been used during the Run 2001 almost exclusively to correct the residual coupling in the machine, after the local correction in the IR triplets (see Section 2). It was demonstrated that the system could be used to decouple that machine to the tune measurement system precision of $\sim 0.001-0.0005$ for tune meter and HF Schottky [5]. The latter were the tune measurement systems routinely used for decoupling operations. The PLL use for decoupling, potentially providing a much higher resolution $\left(\sim 10^{-5}\right)$, will be discussed in Section 5 .

\begin{tabular}{|l|l|l|}
\hline configuration & method & $\Delta \boldsymbol{Q}_{\min }$ \\
\hline Systematic a1(-4 units) in all dipoles & Model & 0.050 \\
\hline 1 Q2 triplet quadrupole tilted by 1 mrad & Model & 0.017 \\
\hline Random roll of 0.5mrad in all quadrupoles(average) & Model & 0.058 \\
\hline Random roll of $0.5 \mathrm{mrad}$ in all quadrupole (worst seed) & Model & 0.1312 \\
\hline Random roll of 0.5 mrad in all triplet quadrupoles & Model & 0.043 \\
\hline Systematic a1 (1 unit) in one DX IR dipole & Model & 0.0003 \\
\hline Systematic a1 (1 unit) in one DO IR dipole & Model & 0.0015 \\
\hline 1 skew family powered at kl=0.0005 m & -1 & 0.024 \\
\hline $\begin{array}{l}\text { Maximum skew family corrector strength } \\
\text { (3 families powered ae 50A, kl=0.001785 } \mathrm{m}^{-1}\end{array}$ & Model & 0.197 \\
\hline Uncorrected $\Delta Q_{\min }$ Blue ring, 2000 run $\left(2.5 \mathrm{~m} \beta^{*}\right.$ lattice) & Model & \\
\hline Uncorrected $\Delta Q_{\min }$ Blue ring, 2001 run $\left(10 \mathrm{~m} \beta^{*}\right.$ lattice) & Data 2000 & 0.03 \\
\hline & Data 2001 & 0.01 \\
\hline
\end{tabular}

Table 6. Comparison of coupling effects in terms of the resulting $\Delta Q_{m i}$

Although the ultimate correction quality in 2001 has been to $\Delta Q_{\min }=0.0005$, it has been our experience that for operations the coupling had to be typically controlled to $\Delta Q_{\min } \sim 0.005$. Global coupling corrections were performed at injection and flattop. Global decoupling readjustment was required after machine retuning larger than typically 0.01 (as the global correction is valid only in the vicinity of the tunes where the tune scan is performed), and after orbit correction, that typically generate tune shift.

The typical global decoupling system performance after dedicated tune and family scans are summarized in Table 7 and 8, respectively for the gold and proton run.

\begin{tabular}{|l|c|c|c|c|}
\hline \multirow{2}{*}{} & \multicolumn{3}{|c|}{ Au Run } & \\
\cline { 2 - 5 } & Blue & Yellow & Blue & Yellow \\
\hline Configuration & Injection & Injection & Store & Store \\
\hline
\end{tabular}




\begin{tabular}{|c|c|c|c|c|c|}
\hline \multicolumn{2}{|l|}{$\Delta$ Qmin after correction } & 0.0005 & 0.006 & 0.004 & 0.0005 \\
\hline Skew Quad & $\# 1$ & 0 & 0 & 0 & -0.00005 \\
Family & $\# 2$ & 0.0006 & -0.0005 & 0.0001 & -0.0005 \\
Settings (kL) & $\# 3$ & 0 & -0.0001 & 0 & 0.0005 \\
\hline Set Tunes & $\mathrm{x}$ & 28.4 & 28.459 & 28.1335 & 28.213 \\
& $\mathrm{y}$ & 29.225 & 29.199 & 29.217 & 29.096 \\
\hline Measured Tunes & $\mathrm{x}$ & 28.2207 & 28.228 & 28.208 & 28.2169 \\
& $\mathrm{y}$ & 29.2202 & 29.234 & 29.2124 & 29.2164 \\
\hline Tune measurement system & & Artus FFT & Artus & Artus FFT & HF Schottky \\
\hline Date recorded & $7 / 12 / 2001$ & $7 / 2 / 2001$ & $10 / 12 / 2001$ & $11 / 16 / 2001$ \\
\hline
\end{tabular}

Table 7. Global decoupling system performance after dedicated scans (gold run).

\begin{tabular}{|c|c|c|c|c|}
\hline & \multicolumn{3}{|c|}{ PP Run } \\
\hline & & Blue & Yellow & Yellow \\
\hline \multicolumn{2}{|l|}{ Configuration } & Injection & Injection & Store \\
\hline \multicolumn{2}{|l|}{$\Delta$ Qmin after correction } & 0.0005 & 0.001 & 0.001 \\
\hline Skew Quad & \#1 & 0.0007 & -0.0001 & 0.0002 \\
\hline Family & \#2 & -0.00005 & -0.00006 & 0 \\
\hline Settings (kL) & $\# 3$ & 0.00005 & 0.00006 & 0.0003 \\
\hline \multirow[t]{2}{*}{ Set Tunes } & $\mathrm{x}$ & 28.313 & 28.413 & \\
\hline & $\mathrm{y}$ & 29.173 & 29.034 & \\
\hline \multirow[t]{2}{*}{ Measured Tunes } & $\mathrm{x}$ & 28.227 & 28.228 & 28.213 \\
\hline & $\mathrm{y}$ & 29.2265 & 29.227 & 29.214 \\
\hline \multicolumn{2}{|c|}{ Tune measurement system } & Artus FFT & Artus FFT & Artus FFT \\
\hline \multicolumn{2}{|l|}{ Date recorded } & $12 / 18 / 2001$ & $12 / 18 / 2001$ & $1 / 18 / 2002$ \\
\hline
\end{tabular}

Table 8. Global decoupling system performance after dedicated scans (proton run).

Coupling correction during Run 2001 was done exclusively at injection and flattop, with the injection settings propagated during ramping, as the method relies on scanning tunes and skew quadrupoles, not possible in a dynamic situation. Alternative correction methods that allow dynamic correction will be discussed in Section 5. The skew quadrupole family settings over the life of Run 2001 (both Au and PP) at injection and storage respectively are shown in Figure 10.

Figure 11 shows a typical tune scan to bring the tunes together, in this example at flattop in Yellow, during the polarized proton run. Tune meter measurements agree well with HF Schottky measurements, once the time lag in the latter due to averaging is taken into consideration. 
Global coupling skew quadrupole correctors

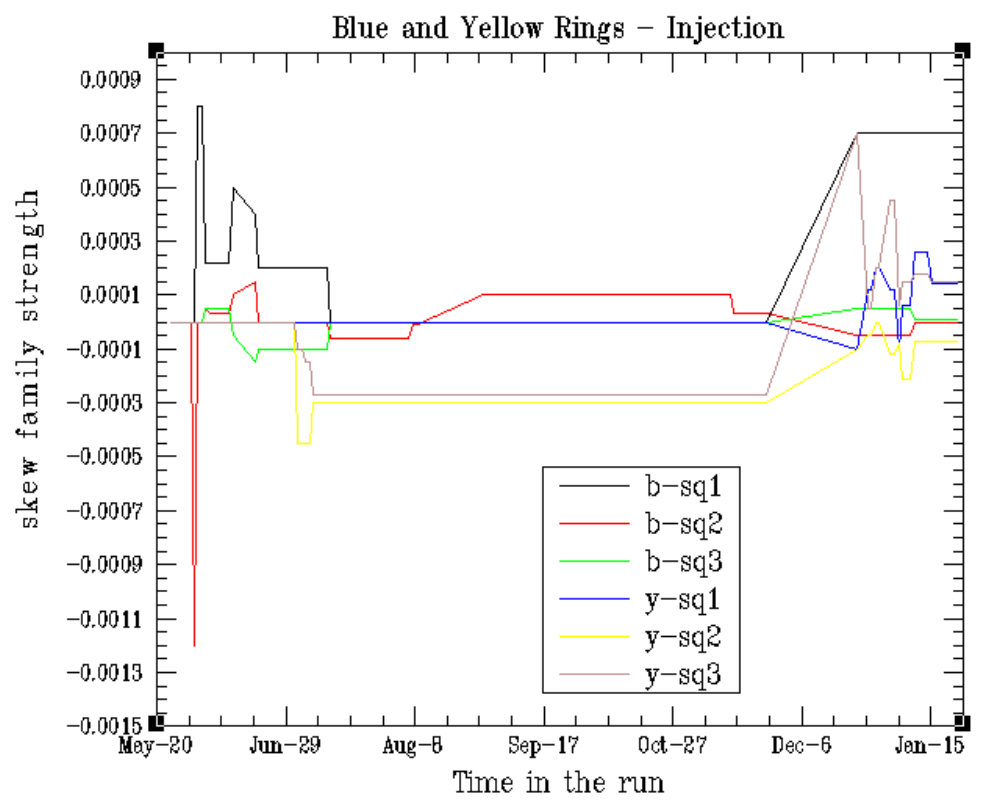

\section{Global coupling skew quadrupole correctors}

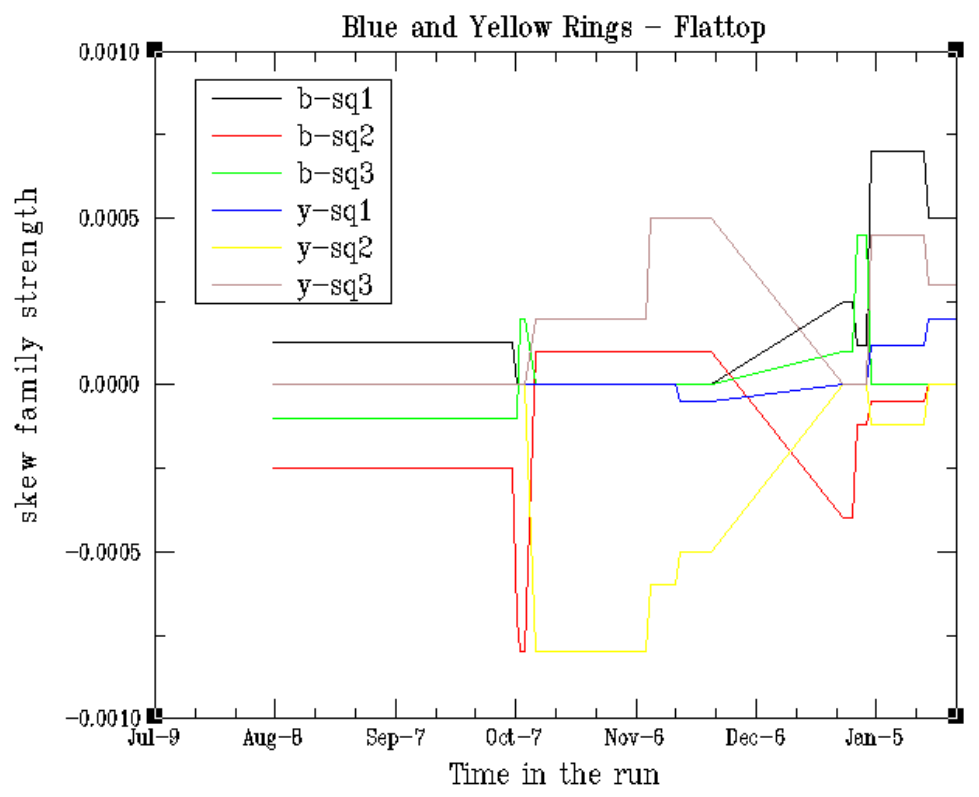

Figure 10. Global skew quadrupole strengths during run 2001, injection and flattop. 


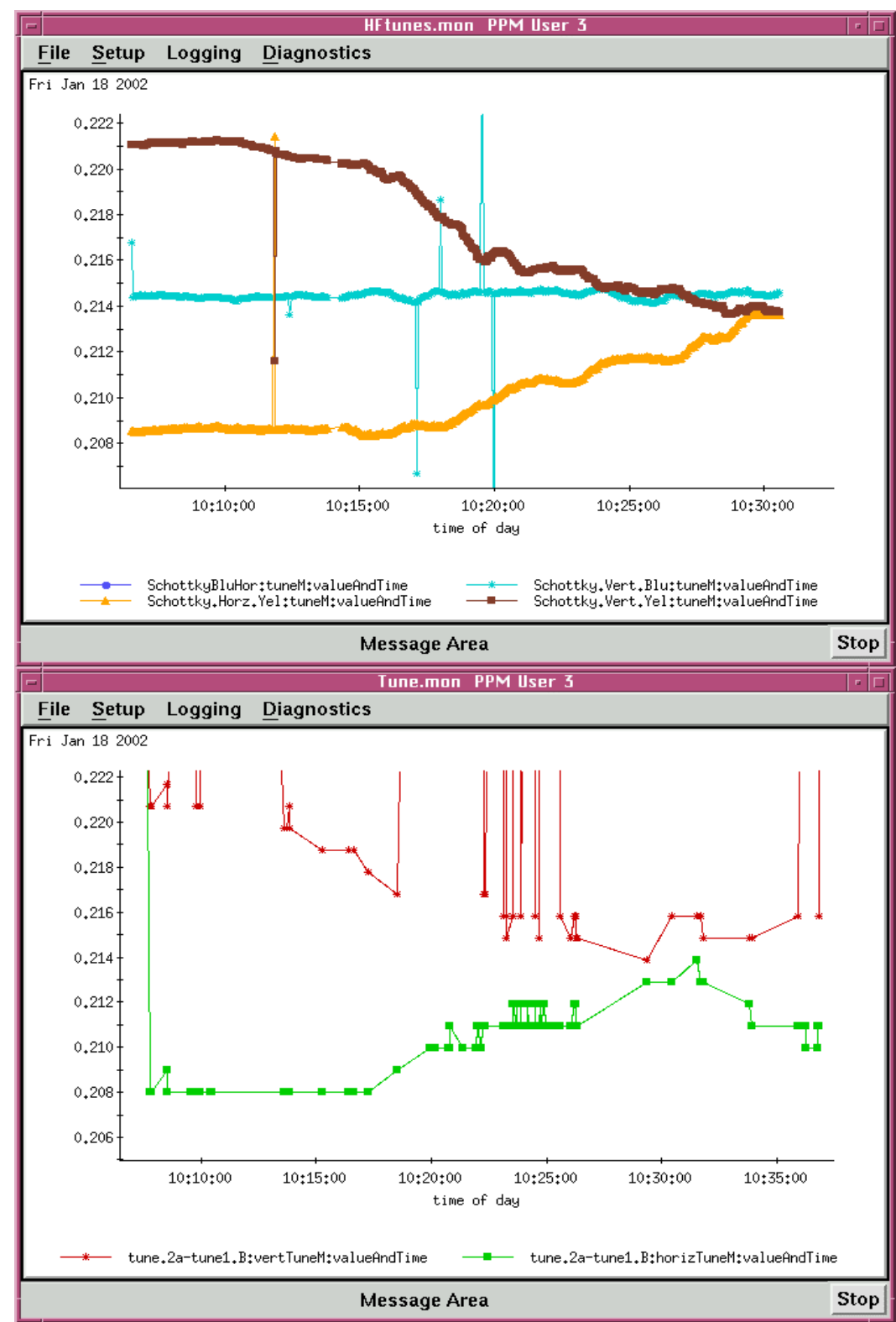

Figure 11. Tune as measured by HF Schottly and the tune meter during a global decoupling scan (flattop, polarized protons, yellow ring)

\subsection{Global decoupling application package}

An application package (DQMIN) has been developed for global linear decoupling during run 2001. It consists of a series of TCL scripts, able to interface with the tune measurement systems and the RampEditor for tune and skew quadrupole family control. In more detail, the script functionality include:

- $\quad$ Setting of the desired tunes

- Monitoring magnet current changes

- Getting measured tunes from the existing tune measurement systems (Artus, PLL and HF Schottky

- Visualization of tune scans vs. set-tunes with separate displays for set- $v_{\mathrm{x}}$ and set- $v_{\mathrm{y}}$.

- Setting of skew quadrupole family strengths 
- Visualization of tune scans vs. skew quads family strengths with displays for all the three families.

The application is available in StartUp. Documentation about the scripts and instructions to perform global decoupling with the DQMIN application package can be found in Appendix 1. The same information is also available online at http://www.cadops.bnl.gov/RHIC/setup/dqmin.html.

\section{Measurement of the linear coupling resonance}

Linear coupling is one of the factors that can affect the lifetime in RHIC. As explained in Section 3, the traditional minimum tune separation method requires a tune scan and cannot be used either parasitically or during the ramp. A new technique of measuring the linear coupling resonance was developed at CERN [6] that quantifies the degree of coupling by comparing the secondary fundamental tune line to the primary one. The very presence of the secondary line is a signature of coupling. The advantage of this method is that it works without changing the machine operating working point. This method can also localize the coupling source by mapping out the coupling driving terms through the ring. Local information is particularly useful in the interaction regions. A beam experiment with the aim of measuring linear coupling was performed during the 2001 polarized proton run. The RHIC tune meter kickers were used to kick beam in both planes and 1024 turn-by-turn (TBT) beam position data were recorded around the ring. We will discuss theory, experiment and results respectively in Section 4.1 to 4.3 below.

\subsection{The method}

The skew quadrupole component in an accelerator drives the coupling resonance at:

$$
Q_{x} \pm Q_{y}=p
$$

where $Q_{x}$ and $Q_{y}$ are the tunes of the horizontal and vertical betatron oscillation and $p$ is an integer. According to the Normal Form theory, in the presence of coupling, the coupling resonance $(1,1)$ corresponds to the $(0,1)$ spectral line in the horizontal Fourier spectrum, and appears as $(1,0)$ in the vertical Fourier spectrum. Hence, the strength of the coupling resonance can then be characterized with the amplitude of the $(0,1)$ line in the horizontal spectrum or the $(1,0)$ line in the vertical spectrum, normalized by the amplitude of the base spectral line of the corresponding plane.

In general, this method requires turn-by-turn beam position data in both planes around the accelerator. The normalized momentum $p_{x, y}$ can be derived from the beam position data from two BPM's separated by a phase advance of about 90 degrees. This method was first tested at the CERN SPS during 2000 and 2001 in a resonance driving terms experiments dedicated to the compensation of the SPS linear coupling. The amplitude ratio of the horizontal $(0,1)$ and $(1,0)$ lines was measured as a function of the current settings of only one SPS skew quadrupole family. The minimum of this ratio was determined and the corresponding minimum tune split $\Delta Q_{\min }$ was measured as $2 \times 10^{-4}$.

An offline code has been developed at CERN to calculate the drive terms of the coupling. [7] The code first takes two pairs of BPM' s, one horizontal and one vertical, and calculates the Fourier spectrum of each beam position signal. The code then calculates the amplitude and phase of the spectral line at the position of betatron oscillation frequency. From the amplitude ratio and phase difference between the two BPM's, one can then calculate the normalized coordinate at the location of the BPM. The Fourier spectrum is calculated by the SUSSIX code, which is essentially a high precision Fourier analysis tool.

\subsection{Experiment setup and data analysis}

This method was tried out in RHIC to measure the coupling driving term during run 2001. We used the 2 tune-meter kickers to excite coherent oscillations in both transverse planes simultaneously. In order to obtain significant coherent oscillation, the tune-meter kickers were configured to kick the beam multiple times at a frequency close to the betatron frequency. Each RHIC arc quadrupole has a BPM nearby in the quadrupole focal plane. The phase advance between each two adjacent horizontal (or vertical) BPM' $s$ is about 85 degree. Dual plane BPM's are only available in the interaction regions where the phase advance between the two BPM' $s$ is no longer close to 90 degree. In general, all the RHIC BPM' s are capable of acquiring 1024 turns of beam position data. Some BPM' s, however, were not available during the 
experiment. Turn-by-turn data at all the available BPM' s around the ring were taken with different skew quadrupole family settings. An offline code $[\mathrm{HH}]$ was then used to convert the 1024 beam position data to normal form coordinates.

$$
\begin{aligned}
& x_{i}=x_{i} \\
& x p_{i}=\sqrt{\frac{\beta_{i}}{\beta_{j}}} \frac{x_{i}}{\sin \psi_{i j}}-x_{i} \frac{\cos \psi_{i j}}{\sin \psi_{i j}}
\end{aligned}
$$

Here $x_{i, j}$ are the positions at the two BPM's, $\beta_{i, j}$ the beta functions at each BPM, and $\psi_{i j}$ the phase advance between the two BPM' s. These data were then fed into SUSSIX, a computer code for frequency analysis, to calculate the Fourier spectrum. The amplitude ratio of the $(0,1)$ spectral line to the $(1,0)$ line in the horizontal plane, and the amplitude ratio of the $(1,0)$ line to the $(0,1)$ line in the vertical plane were then computed. Figure 12 shows the measured $(1,1)$ amplitude and $(1,-1)$ at all the BPM' $\mathrm{s}$ for different skew quadrupole settings.
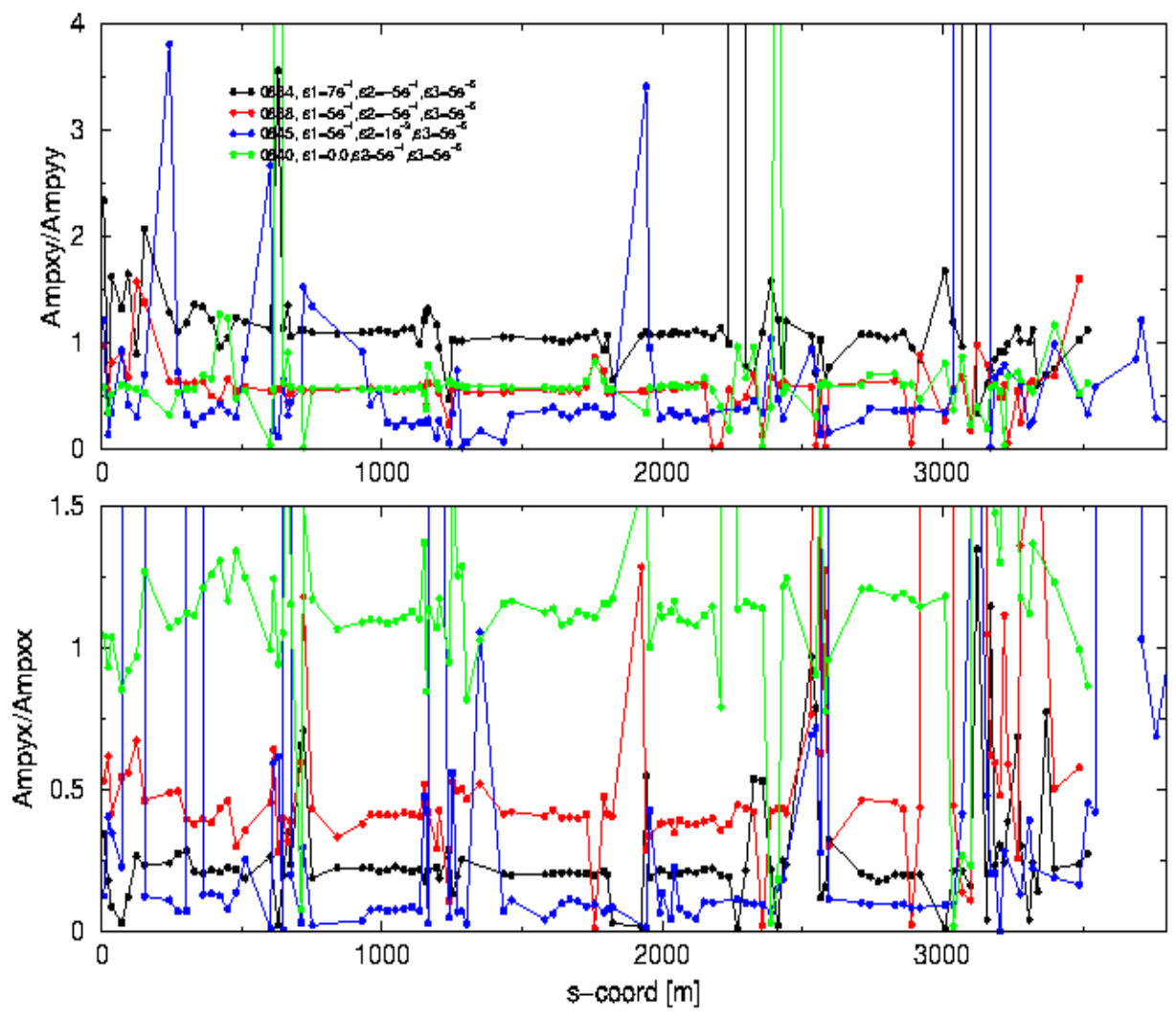

Figure 12. The top plot is the measured $(1,1)$ resonance strength as a function of the BPM position. Four sets of data were taken for different skew quadrupole settings. (the data around the interaction regions are noisier due to the fact that the phase advance between two adjacent BPM's is not close to 90 degrees, fact that reduces the accuracy of the derived normalized complex coordinates)

In general, the amplitude ratio should be a less than 1 . The fact that some sets of the data are actually larger than 1 could be explained by the fact that coherence in these cases is mainly coming from the other plane, induced by coupling. The in plane coherence induced by the tune-meter kicker de-coheres quickly because of large chromaticity. When this happens, the ratio of the $(0,1)$ line and $(1,0)$ line becomes larger than 1 . This method can also be used to measure local coupling. In general, the sudden change of amplitude ratio between different locations in the ring represents a local coupling source. This can be useful for further investigation the local coupling in the RHIC IR's. 


\section{Developments and plans for Run 2003}

The planning of the RHIC Run 2003, to start in November 2002, was initiated at the RHIC Retreat in March 2002. New requirements on the performance of the coupling system emerged, and a plan to meet these requirements is being developed.

Operational experience from 2001 stressed that we need faster coupling correction capabilities, so that decoupling operations can be routinely done every time a closed orbit correction is done, or a change in machine tunes or a change in optics configuration. For example, a change of experiment magnet settings at flattop, typically affects the coupling as the experiments have axial or solenoid fields, so it would be useful to have a 'library' of correction configuration. A faster global correction with the application scripts can be achieved by speeding up the underlying tune measurements (i.e. faster averaging for the HF Schottky at flattop or use of the PLL for decoupling, following the LEP experience) and by speeding up the skew quadrupole scans (for instance by providing the PS control software with knobbing capabilities).

Another requirement on coupling correction capabilities comes from tune control on the ramp. Tune control during ramping is fundamental to minimize beam losses on resonances in general, and to preserve polarization during proton operations. Tune feedback is required to keep the tunes constant on the ramp, and optimal PLL operation require coupling minimization, unless the PLL horizontal and vertical tune signals are separated in frequency or time domain. Operationally, during the commissioning of PLL in 2001, we set the tunes artificially apart at injection by $\sim 0.02$, and that typically prevented losing the lock because of coupling effects on the ramp. However, when tune feedback will be used in regular operations, we have to be able to set the tunes on operational requirements other than coupling minimization, hence coupling control on the ramp is desirable.

The minimum tune separation technique is obviously not a good candidate, because is time consuming and changes the tunes, both incompatible with a dynamic situation. Work has started on devising methods to correct coupling that can work on the ramp.

Table 9 summarizes the techniques that were discussed at the RHIC Retreat. We will describe in the following their capability, experimental experience where it applies, possible implementation and use in RHIC for Run 2003.

\begin{tabular}{|l|l|l|l|l|}
\hline METHOD & measure & correction & On the ramp & global/local \\
\hline$\Delta$ Qmin & indirect & Yes & No & Global \\
\hline IR bumps & Yes & Yes & No & Local (IR) \\
\hline Action-Phase jump & Yes & Possible & Yes & Local (IR) \\
\hline $\begin{array}{l}\text { SUSSIX method } \\
\text { (resonance compensation) }\end{array}$ & Yes & Yes & Yes & $\begin{array}{l}\text { Global correction } \\
\text { Local measure }\end{array}$ \\
\hline N-turn transfer matrix & Yes & Possible & Yes & Global \\
\hline Teapot local decoupling & Yes & Yes & Yes & Local \\
\hline Schottky line & Yes & Yes & Yes & Global \\
\hline
\end{tabular}

Table 9. Summary of coupling measurement and correction techniques

As previously discussed, the first three methods in Table 9 were used in operation during Run 2001, and we will retain the capability of setting the local skew IR correctors and that of performing minimum tune approach if necessary at injection and flattop.

The (SUSSIX) coupling resonance compensation was described in Section 4. The advantage of the method is that it does not require moving the base tunes, so it is a good candidate to allow coupling correction on the ramp. Kick and acquisition of turn-by-turn data can be done on the ramp (i.e. on ramp step-stones) and from the analysis of the ramp data, corrections can be feed-forwarded to the next ramp. The plan for 2003 is to have an application available on line that allows easy acquisition and quick analysis of turn-by-turn data. 
Another technique on the drawing board is based on the computation of the N-turn transfer matrix. Information about the global coupling of the machine can be in principle inferred by the off-diagonal terms of the 1-turn transfer matrix

Before decoupling (01-08-2001). 42-turn map.

\begin{tabular}{|l|l|l|l|}
\hline 0.3935 & -1.1215 & $\mathbf{- 1 . 4 5 5 4}$ & $\mathbf{4 . 6 1 8 5}$ \\
\hline-0.00406 & 0.3822 & $\mathbf{- 0 . 0 8 3 7}$ & $\mathbf{- 0 . 0 8 6 5}$ \\
\hline $\mathbf{0 . 5 3 1 9}$ & $\mathbf{- 1 3 . 7 9 4 5}$ & 0.2947 & 1.5399 \\
\hline $\mathbf{- 0 . 0 1 4 6}$ & $\mathbf{0 . 9 4 8 0}$ & -0.0056 & 0.1527 \\
\hline \multicolumn{4}{|c|}{ After decoupling (01-09-2001). 123-turn map. } \\
\hline-0.0676 & 3.0765 & $\mathbf{1 . 8 5 0 7}$ & $\mathbf{2 . 6 6 6 0}$ \\
\hline-0.0056 & 0.1656 & $\mathbf{0 . 0 8 3 4}$ & $\mathbf{0 . 3 9 6 8}$ \\
\hline $\mathbf{0 . 0 9 6 1}$ & $\mathbf{3 . 0 0 9 1}$ & -0.1847 & 0.9820 \\
\hline $\mathbf{- 0 . 0 5 6 3}$ & $\mathbf{1 . 0 0 7 3}$ & -0.0137 & -0.2917 \\
\hline
\end{tabular}

Table 10. Comparison of N-turn matrices before and after decoupling.

In practice though the off diagonal terms at RHIC are typically $10^{-4}$ smaller than the diagonal ones, unfavorable for experimental robust determination. However, it can be demonstrated that for the N-turn map, where $\mathrm{N}$ is half the coupling beat period, the off diagonal are the same order than the diagonal terms. Table 10 shows the comparison of the N-turn map for RHIC (Run 2001 data), before and after a global decoupling operation (with the $\Delta \mathrm{Q}_{\min }$ method). By modeling the predicted changes of the off diagonal terms of the N-turn, this technique can be used for global coupling corrections.

Another method that has the capability of measuring (and correcting) coupling on the basis of turn-by-turn BPM data is the local decoupling technique. A detailed derivation of the method can be found in [8] and the study and modeling of its application to RHIC in [9]. The essence of the method is to use the turn-byturn signal measured at all double plane BPM's in the machine. After kicking (or exciting) the beam in one plane, it is possible to extract from the turn-by-turn data the ratio between the out of plane and in plane oscillations. From this it is possible to derive the magnitude of the eigen-angles, a measure of coupling, locally, at every double plane BPM. Furthermore, it is possible from the data to build a badness function, function of the skew quadrupole corrector strengths available in machine. Minimization of the badness function gives the skew quadrupole strengths to correct the coupling locally. This method gave excellent results in simulation for RHIC (the correction algorithm is implemented in the Fortran version of the Teapot code). The plan for run 2003 is to implement the local decoupling algorithms first in the present RHIC offline model (based on the UAL software package), then make it available in the upgraded online machine model.

\section{Acknowledgements}

The authors would like to thank their colleagues M. Blaskiewicz, P. Cameron, S. Peggs, T. Satogata, T. Roser for fruitful discussions and suggestions concerning coupling control at RHIC, as well as F. Schmidt and R. Tomas from CERN for their collaboration in the coupling resonance beam experiment.

\section{References}

[1] V.Ptitsyn, J. Cardona, F. Pilat, J.P Koutchouk, "Measurement and Correction of Linear Effects in the RHIC Interaction Regions", PAC 2001, Chicago.

[2] F. Pilat, P. Cameron, V. Ptitsyn, J-P. Koutchok, "Linear and Nonlinear Corrections in the RHIC Interaction Regions", EPAC 2002, Paris. 
[3] J. Cardona, S. Peggs, F. Pilat, V. Ptitsyn, T. Satogata, "Determination of Linear and Nonlinear Components in RHIC Interaction regions from Difference Orbit Measurements", EPAC 2002, Paris.

[4] J. Cardona, Ph.D. Thesis, Stony Brook University, 2003 (To be published)

[5] F. Pilat, J. Beebe-Wang, W. Fischer, V. Ptitsyn, T. Satogata, "Coupling Analysis and Correction at RHIC", EPAC 2002, Paris.

[6] R. Bartolini, F. Schmidt, "Normal Form via Tracking or Beam Data", LHC Project Report 132, 1999

[7] R.Tomas, Private Communication

[8] R.Talman. "An universal algorithm for accelerator correction", Proceedings of the Advanced Beam Dynamics Workshop on effects of errors in accelerators, their diagnosis and correction", Corpus Christi, October 1991.

[9] F.Pilat "Correction of the triplet skew quadrupole errors in RHIC", RHIC/AP/58, March 1995

\section{Appendix 1. Guide for using the DQMIN application package for global decoupling}

\section{A1. Open the application}

From the StartUp operation menu, select the method (PLL, Schottky, or ARTUS) and ring (blue or yellow) DQMIN script. The application window will show up on the screen by clicking once on one of the following applications from the list:

- Decoupling (ARTUS, Blue

- Decoupling (ARTUS, Yellow)

- Decoupling (PLL, Blue)

- Decoupling (PLL, Yellow)

- Decoupling (Schottky, Blue)

- Decoupling (Schottky, Yellow)

Do not open the application, then use it during a ramp or after a ramp. It should be opened and used at an injection or at a store alone. You MUST RESTART the application if there has been a ramping-up or a ramping-down.

\section{A2. Use the application}

1. The values shown in the box "SET TUNE" are the set tunes at the time when the application is opened. To change the set tunes, enter the desired values in the "muX set" AND "muY set" entryboxes in "SET TUNE", then push "Enter" on the keyboard while cursor is in one of the entryboxes. You will see a short time period that the entry boxes being blank which allows magnet current becoming stable.

2. If you have chose PLL or Schottky application, the measured tunes are shown and updated automatically in "MEASURED TUNE" box provided PLL or Schottky instrumentation is tracking the tunes. If you have chose ARTUS application, you can push "Enter" on the keyboard while cursor is in one of the entry-boxes in "MEASURED TUNE" box in order to trigger tune measurement. 
3. The values shown in the box "SET SKEW STRENGTHS" are the skew quad family strengths at the time when the application is opened. To change the strengths, enter the desired values in ALL entry-boxes in "SET SKEW STRENGTHS", then push "Enter" on the keyboard while cursor is in one of the entry-boxes. You will see a short time period that the entry boxes being blank which allows magnet current becoming stable.

4. Caution: all the values are expected in the box "SET TUNE" or "SET SKEW STRENGTHS" when you make tune or skew quad family setting changes. Do not leave one of the entry box blank.

5. All the tune scan graphs are on auto-scale. Holding the left button on the mouse to zoom-in. Push the right button on the mouse to return to auto-scale.

\section{A3. Decoupling procedure}

1. Record the current tune settings.

2. SCAN OF MEASURED-TUNES VS. SET-TUNES:

Do a tune scan around the current set tunes. The goal is to bring the measured horizontal and vertical tunes together by changing the set tunes.

\section{Suggestion:}

a) Move the higher tune down and the lower tune up by changing set tunes by 0.003 0.005 at the same time. If a tune is close to a resonance, only move 0.001-0.002 at each step.

b) Watch "qmain" and "qtrim" boxes in "WFG MAIN BUS" box to see if the changes of the magnet current are completed.

c) If measure tunes look reasonable, click on "Add data point" button on both pictures on the top row.

d) Repeat step a) to c) until you see the minimum horizontal and vertical tune separation.

e) If you need to start over the tune scan, click on "Clear all data point" buttons.

3. Minimize the measured horizontal and vertical tune separation by putting the set-tunes at the value where horizontal and vertical tune separation reached minimum in 1).

4. SCAN OF MEASURED-TUNES VS. SKEW QUAD STRENGTHS Do a tune scan around the current skew quad family settings. The goal is to further bring the measured horizontal and vertical tunes together by changing the skew quad family settings.

\section{Suggestion:}

f) Move the skew quad family \#1 by 0.00005-0.0002 at each step.

g) If measure tunes look reasonable, click on "Add data point" button on the first pictures on the bottom row.

h) Repeat step f) and g) until you see the minimum horizontal and vertical tune separation.

i) Minimize the measured horizontal and vertical tune separation by putting the skew quad family $\# 1$ at the value where horizontal and vertical tune separation reached minimum.

j) Then do tune scan with family $\# 2$ and $\# 3$ as step f) through i). At each step change the two families have opposite values. (For example: Family $\# 2=0.0001$, Family $\# 3=-$ $0.0001)$.

5. Put the set tunes back to the original settings recorded in 1).

6. If you are satisfied with the result and want to save the settings for the later ramps, put the final skew quad family settings into "RAMP EDITOR".

Please forward requests or report bugs to Joanne Beebe-Wang (344-3646 bbwang@bnl.gov) 